\title{
A Conservative and Monotone Characteristic Finite Element Solver for Three-Dimensional Transport and Incompressible Navier-Stokes Equations on Unstructured Grids
}

\author{
Bassou Khouya ${ }^{1,2}$, Mofdi El-Amrani ${ }^{2, *}$ and Mohammed Seaid ${ }^{3}$ \\ 1 International Water Research Institute, University Mohammed VI Polytechnic, \\ Benguerir, Morocco. \\ 2 Mathematics and Applications Laboratory, FST, Abdelmalek Essaadi University, \\ Tangier, Morocco. \\ ${ }^{3}$ Department of Engineering, University of Durham, South Road, Durham DH1 \\ 3LE, UK.
}

Received 17 November 2020; Accepted (in revised version) 13 September 2021

\begin{abstract}
We propose a mass-conservative and monotonicity-preserving characteristic finite element method for solving three-dimensional transport and incompressible Navier-Stokes equations on unstructured grids. The main idea in the proposed algorithm consists of combining a mass-conservative and monotonicity-preserving modified method of characteristics for the time integration with a mixed finite element method for the space discretization. This class of computational solvers benefits from the geometrical flexibility of the finite elements and the strong stability of the modified method of characteristics to accurately solve convection-dominated flows using time steps larger than its Eulerian counterparts. In the current study, we implement three-dimensional limiters to convert the proposed solver to a fully mass-conservative and essentially monotonicity-preserving method in addition of a low computational cost. The key idea lies on using quadratic and linear basis functions of the mesh element where the departure point is localized in the interpolation procedures. The proposed method is applied to well-established problems for transport and incompressible Navier-Stokes equations in three space dimensions. The numerical results illustrate the performance of the proposed solver and support its ability to yield accurate and efficient numerical solutions for three-dimensional convection-dominated flow problems on unstructured tetrahedral meshes.
\end{abstract}

AMS subject classifications: 65M25, 65N30, 65Z05, 35Q30

Key words: Mass-conservative, monotonicity-preserving, modified method of characteristics, finite element method, convection-dominated problems, incompressible Navier-Stokes equations.

*Corresponding author. Email addresses: khouya.bassou@um6p.ma (B. Khouya), mofdi.elamrani@urjc.es (M. El-Amrani), m.seaid@durham.ac.uk (M. Seaid) 


\section{Introduction}

Transport in incompressible flows takes place in many applications in science and engineering. This class of problems occur in a many applications in nature and technology, for example in the simulation of a heat transport in draining films [45], groundwater flows in soils [25], and the transport of ferro-fluids under magnetic fields [4] among others. Developing robust numerical solvers for this set of problems is still challenging in the situation of convection-dominated flows for which convection terms are manifestly more important than the diffusion terms particularly if some nondimensional parameters attend high values. As example of these parameters we mention the well-known Reynolds number for the incompressible Navier-Stokes equations and the Peclet number for the convectiondiffusion equations. There exist many numerical techniques in the literature to solve the transport and incompressible Navier-Stokes equation. In case of convection-dominated flows, the conventional Eulerian finite element methods use up-stream weighting in their implementations to stabilize the discretization. For example, the most popular Eulerian finite element methods are the streamline upwind Petrov-Galerkin methods $[3,5,11]$, the Taylor-Galerkin methods $[8,12,16]$ and the Galerkin/least-squares methods $[3,9,26]$. However, truncation errors generated by the time integration in these conventional Eulerian methods are dominant and require Courant-Friedrichs-Lewy (CFL) stability conditions which impose sever restrictions on the time steps used in the numerical computations. Eulerian numerical methods for three-dimensional advection-diffusion problems have also been investigated in [10,13, 27, 35, 48] among others. In [13], a simple comparison between implicit and explicit finite difference methods have been studied for a class of linear three-dimensional advection-diffusion problems with constant coefficients. However, all results presented were in Cartesian meshes which restrict their application to simple regular domains. High-order compact finite difference methods have also been proposed in [48] for the stationary semi-linear three-dimensional advectiondiffusion equations. Eulerian-based methods for the three-dimensional incompressible Navier-Stokes equations have also been discussed in [10,14,15,32,33,36,41,43,44] among others. A dimension split method has been studied in [10] and a multi-stage Rosenbrock scheme has been applied to the three-dimensional incompressible Navier-Stokes equations in [14]. However, these methods fail to resolve flow structures at high Reynolds numbers. In [44], a multigrid adaptive unstructured finite element method has been proposed for the numerical solution of the three-dimensional incompressible Navier-Stokes equations. However, the adaptation process in this scheme requires assembling matrices at each time step which increases the computational cost. A compact mixed finite element method has been proposed in [43] to reduce the computing time for solving linear algebraic equations resulted from the discretization of three-dimensional incompressible Navier-Stokes equations but this study dealt with steady problems only. In [32,33], a class of finite difference schemes have been implemented for space discretization of threedimensional incompressible Navier-Stokes equations. However, the main drawback of these methods is that they are not able to resolve complex flow problems in irregular 
geometries. Numerical simulation of three-dimensional incompressible flows in parameterized pipes has been performed in [15]. Spectral collocation methods have also been studied in [41] for three-dimensional incompressible Navier-Stokes equations with variable density. To avoid the instability problems, authors in [36] proposed a high-order implicit preconditioned finite difference method to solve the three-dimensional incompressible flows using the pseudo-compressibility approach. However, restrictions related to the solution of nonlinear systems and Cartesian geometry decrease the efficiency of this method.

Semi-Lagrangian methods employ the modified method of characteristics and have been widely used in the literature to solve several problems in physical and engineering applications. Indeed, semi-Lagrangian finite element methods have been used for example in [24] for two-dimensional convection-diffusion problems, in [22] for twodimensional incompressible Navier-Stokes equations, in [21] for tidal flows, and in [19] for two-dimensional natural and mixed convection flows. The central idea in these semiLagrangian finite element methods lies in reformulating the governing equations using the Lagrangian coordinates defined by the characteristic curves associated with the considered problem. The time derivative and the advection term are combined in the total derivative as a directional derivative along the characteristics which can be viewed as a characteristic time-stepping algorithm. Therefore, the Lagrangian treatment in these methods greatly reduces the time truncation errors in the Eulerian methods, see for instance $[18,20]$. In addition, semi-Lagrangian methods allow for higher time steps exceeding those permitted by the CFL stability condition in its Eulerian finite element counterpart for the convection-dominated flows. A variety of semi-Lagrangian methods has also been recently studied for three-dimensional problems in $[28,29]$ among others. Although these methods are unconditionally stable, they suffer from the lack of preserving the maximum/minimum principles and failure to guarantee the conservation properties. Many research studies have been carried out in the literature to reconstruct conservative semi-Lagrangian finite element methods. For instance, remapping the Lagrangian volume procedure using the cubic-interpolated propagation techniques has been proposed in [46]. However, the mass in this method is considered as an additional variable and it is used in a correction step. For solving the Vlasov-Poison system in collision-less plasma applications, a conservative semi-Lagrangian method has also been introduced in [6]. The conservation in this method is guaranteed by a dimensional splitting using the fifth-order finite difference Hermite weighted interpolation accounting for the WENO limiters to control oscillations. However, this approach may become computationally very demanding for realistic applications in convection-dominated flows. Using the adjoint property to reconstruction fully conservative methods has been investigated in [7] among others. This method uses the concept of support operators proposed in [49] for which the adjoint property between the continuity and advection equations is used along with a column-balance property to enforce the conservation property for an arbitrary advection method. However, for the case of convection-dominated flow problems 
on unstructured grids, this method may become unstable. In [34] a time-splitting Fourier spectral method has been proposed for solving the semi-classical Schrödinger equation in electromagnetic applications. The main focus in this method is on the non-uniform fast Fourier transform algorithm to interpolate Fourier series in the convection step but the conservation property is ensured using a high-order interpolation procedure but considering periodic boundary conditions only.

The aim of the present work is to develop a robust computational method to accurately approximate numerical solutions of the three-dimensional transport and incompressible Navier-Stokes problems on unstructured tetrahedral meshes. The main objective is to implement a fast and accurate characteristic finite element method that satisfies both monotonicity and conservation properties at each time step in the computational process. The proposed characteristic finite element method can be interpreted as a fractional-step algorithm where the convection and the diffusion parts in the considered problems are solved separately. To guarantee the monotonicity and conservation properties at each time step, we consider limiters by combining the linear and quadratic finite element interpolations. It should be mentioned that a similar monotone and conservative semi-Lagrangian finite element method has been proposed in [2] for solving two-dimensional transport problems. It should be stressed that the main features of the semi-Lagrangian finite element method proposed in the current work are on one hand, the capability to satisfy the monotonicity and conservation properties allowing for numerical solutions free from spurious oscillations, and on the other hand, the achievement of strong stability and high accuracy for numerical solutions with steep gradients. The application of this method for solving three-dimensional incompressible Navier-Stokes equations is also carried out in the current work. The performance of the proposed characteristic finite element method is demonstrated for several test examples of threedimensional transport problems including the benchmark of flow past a circular cylinder. Numerical results presented in this study show high resolution, full conservation and monotonicity properties of the proposed characteristic finite element method, and support the straightforward extension of the method to highly complex, physically based three-dimensional flow problems.

This is structured as follows. The characteristic finite element method for solving the three-dimensional transport problems is formulated in Section 2. This section includes the approximation of the characteristic curves and the implementation for transport equations. Section 3 is devoted to the development of a new mass-conservative and monotonicity-preserving characteristic finite element method. The implementation of the proposed method for solving the incompressible Navier-Stokes equations is presented in Section 4. In Section 5, we demonstrate the numerical performance of the proposed method using different examples of transport and incompressible Navier-Stokes equations. Our new approach is demonstrated to enjoy the expected conservation and monotonicity properties. Conclusions are included in Section 6. 


\section{Characteristic finite element method}

For the formulation of the characteristic finite element method we consider the following pure transport problem

$$
\begin{aligned}
& \frac{D u}{D t}:=\frac{\partial u}{\partial t}+\boldsymbol{v}(x, t) \cdot \nabla u=0, \quad(x, t) \in \Omega \times(0, T], \\
& u(x, 0)=u_{0}(x), \quad x \in \Omega \text {, }
\end{aligned}
$$

where $\Omega$ is a bounded domain in $\mathbb{R}^{3}$ with Lipschitz boundary $\Gamma,(0, T]$ a time interval, $x=$ $(x, y, z)^{\top}$ the position variable, and $\nabla=\left(\frac{\partial}{\partial x}, \frac{\partial}{\partial y}, \frac{\partial}{\partial z}\right)^{\top}$ the gradient vector. In $(2.1), v(x, t)=$ $\left(v_{1}(x, t), v_{2}(x, t), v_{3}(x, t)\right)^{\top}$ is the velocity field and $u_{0}(x)$ is the initial condition. It should be noted that the total derivative $\frac{D u}{D t}$ in (2.1) known also by the material derivative is used to measure change rate of the solution $u$ following the trajectories of the flow particles defined by the characteristic curves. The central idea of the characteristic finite element method lies on imposing a regular grid at the new time step and to backtrack the flow trajectories to the previous time step. At the old time step, the solutions are obtained using interpolation procedures from their known values on the regular grid. Next, we discretize the three-dimensional domain $\Omega$ into a finite set of conforming elements $\mathcal{T}_{j}$ $\left(j=1,2, \cdots, N_{e}\right)$ with $N_{e}$ is the total number of elements. Here, the computational domain $\Omega_{h} \subseteq \Omega$ is the combination of all these finite elements. For the solution of the problem (2.1), the conforming finite element space is defined as

$$
\mathcal{V}_{h}=\left\{u_{h} \in \mathcal{C}^{0}(\Omega):\left.u_{h}\right|_{\mathcal{T}_{j}} \in P\left(\mathcal{T}_{j}\right), \forall \mathcal{T}_{j} \in \Omega_{h}\right\},
$$

where

$$
P\left(\mathcal{T}_{j}\right)=\left\{p(\boldsymbol{x}): p(\boldsymbol{x})=\hat{p} \circ F_{j}^{-1}(\boldsymbol{x}), \hat{p} \in P_{m}(\hat{\mathcal{T}})\right\},
$$

with $P_{m}(\hat{\mathcal{T}})$ is the set of polynomials of degree $\leq m$ defined on the reference element $\hat{\mathcal{T}}$ and $\hat{p}(\boldsymbol{x})$ is a polynomial of degree $\leq m$ defined on the element $\hat{\mathcal{T}}_{j}$. Here, $F_{j}: \hat{\mathcal{T}} \longrightarrow \mathcal{T}_{j}$ is an invertible one-to-one mapping from the physical to the reference elements in the computational domain. To discrete the time domain, the time interval is divided into small subintervals $\left[t_{n}, t_{n+1}\right]$ with stepsize $\Delta t=t_{n+1}-t_{n}$ for $n=0,1, \cdots$. We use the notation $u^{n}$ to denote the value of the solution $u$ at time $t_{n}$. Thus, the finite element approximation of the solution $u^{n}(\boldsymbol{x})$ is given by

$$
u_{h}^{n}(\boldsymbol{x})=\sum_{j=1}^{M} U_{j}^{n} \phi_{j}(\boldsymbol{x})
$$

where $U_{j}^{n}$ are the nodal values associated with $u_{h}^{n}(\boldsymbol{x})$ defined as $U_{j}^{n}=u_{h}^{n}\left(\boldsymbol{x}_{j}\right)$ with $\left\{\boldsymbol{x}_{j}\right\}_{j=1}^{M}$ is the set of solutions at the grid points in $\Omega_{h}$ and $M$ is the number of solution mesh points in the computational domain $\Omega_{h}$. In (2.3), $\left\{\phi_{j}\right\}_{j=1}^{M}$ are the global nodal basis functions 
in $\mathcal{V}_{h}$ characterized by the standard property $\phi_{i}\left(\boldsymbol{x}_{j}\right)=\delta_{i j}$ with $\delta_{i j}$ denotes the Kronecker symbol.

Using the notation introduced above, the characteristic curves $\boldsymbol{X}_{h j}^{n}=\boldsymbol{X}_{h}\left(t_{n} ; \mathbf{x}_{j}, t_{n+1}\right)$ associated with the advection problem (2.1) are calculated for each mesh point $x_{j}, j=$ $1, \cdots, M$ by solving the following initial-value problem

$$
\begin{aligned}
& \frac{d \boldsymbol{X}_{h}\left(\tau ; \boldsymbol{x}_{j}, t_{n+1}\right)}{d \tau}=\boldsymbol{v}_{h}\left(\boldsymbol{X}_{h}\left(\tau ; \boldsymbol{x}_{j}, t_{n+1}\right), \tau\right), \quad \forall \tau \in\left[t_{n}, t_{n+1}\right], \\
& \boldsymbol{X}_{h}\left(t_{n+1} ; \boldsymbol{x}_{j}, t_{n+1}\right)=\boldsymbol{x}_{j},
\end{aligned}
$$

where $X_{h}\left(\tau ; x_{j}, t_{n+1}\right)=\left(X_{h}\left(\tau ; x_{j}, t_{n+1}\right), Y_{h}\left(\tau ; x_{j}, t_{n+1}\right), Z_{h}\left(\tau ; x_{j}, t_{n+1}\right)\right)^{\top}$ is the departure point defined at time $\tau$ of a particle that will reach the mesh point $x_{j}=\left(x_{j}, y_{j}, z_{j}\right)^{\top}$ at time $t_{n+1}$. Note that an accurate approximation of the departure points $\boldsymbol{X}_{h}\left(t_{n} ; \boldsymbol{x}_{j}, t_{n+1}\right)$ is a key to the overall accuracy of the characteristic finite element method. A second-order explicit Runge-Kutta scheme has been used in the literature to approximate the solutions of (2.4) but this method has proven to be not accurate enough to maintain a particle on its curved trajectory, see for example $[18,19]$. In the present study, we consider a secondorder extrapolation based on the mid-point rule to approximate the solution of (2.4) along with an iterative procedure, see $[18,29]$ for more details.

In general, the departure points $\boldsymbol{X}_{h j}^{n}$ do not surely coincide with a nodal point in the computational domain $\Omega_{h}$ and therefore, solutions at the departure points $\boldsymbol{X}_{h j}^{n}$ must be evaluated by interpolation using known values at the mesh points of the host element where the points $\boldsymbol{X}_{h j}^{n}$ are localized. In the current work, this interpolation step is achieved using the finite element basis functions in the host element where the departure points $\boldsymbol{X}_{h j}^{n}$ are allocated. Thus, the finite element method allows for high-order basis functions to be used for the interpolation step without need for explicit interpolation polynomials as in the case of finite difference methods, see for instance $[38,39]$. Hence, the finite element solution $\widetilde{u}_{h}^{n}=u\left(\boldsymbol{X}_{h j}^{n}, t_{n}\right)$ is defined by

$$
\widetilde{u}_{h}^{n}=\sum_{j=1}^{M} \widetilde{U}_{j}^{n} \phi_{j},
$$

where $\widetilde{U}_{j}^{n}$ are nodal solutions evaluated at the departure points $\boldsymbol{X}_{h j}^{n}$ using the finite element interpolation of $u_{h}^{n}(\boldsymbol{x})$. Notice that this procedure requires less computational effort than the projection method from the background Eulerian grid onto the Lagrangian grid as discussed in $[17,22,24]$ among others.

\section{Mass-conservative and monotonicity-preserving procedures}

It is well known that most of high-order interpolation procedures do not satisfy desired numerical properties such as conservation, monotonicity and positivity of the computed 

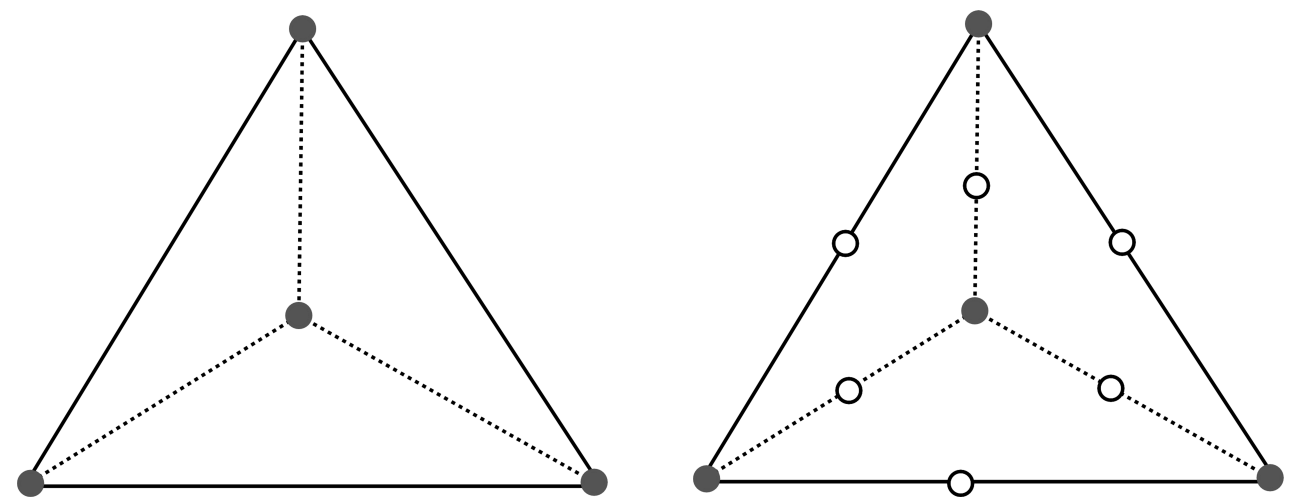

Figure 1: An illustration of the linear $P_{1}$ with 4 nodes (left plot) and the quadratic $P_{2}$ with 10 nodes (right plot) finite elements used in the present study.

solutions. In this study, a limiting procedure is used for the characteristic finite element method to overcome these drawbacks and to ensure that the interpolated solution in a tetrahedral element remains bounded between the maximum and minimum values in the vertices of this element. Therefore, the original method is converted to a nonoscillatory characteristic finite element method at minor additional computational effort. In addition, the reconstructed method highly preserves the shape of the transported fields in the vicinity of steep gradients and it maintains the order of convergence in regions where the solution is sufficiently smooth. Similar techniques have been investigated in $[2,20,21]$ for two-dimensional transport problems. The procedure proposed in the current work uses a limiter between a low-order and a high-order finite element approximations within the host element where the departure points reside. Thus, the linear $P_{1}$ elements and the quadratic $P_{2}$ elements depicted in Fig. 1 are used for the low-order and high-order interpolations, respectively.

Hence, the procedure to evaluate the numerical solution $\widetilde{u}^{n}$ of the transport problem (2.1) is obtained using the following steps:

1. Compute the departure points $\boldsymbol{X}_{h j}^{n}$ by solving the backward differential equations (2.4).

2. Locate using a search-locate algorithm the element $\widetilde{\mathcal{T}}_{j}$ where the departure point $\boldsymbol{X}_{h j}^{n}$ belongs.

3. Calculate the quadratic $P_{2}$ finite element approximation

$$
\widetilde{u}_{H j}^{n}=\sum_{k=1}^{N_{H}} \widetilde{U}_{k}^{n} \varphi_{k}\left(\boldsymbol{X}_{h j}^{n}\right)
$$

where $\left\{\varphi_{1}, \cdots, \varphi_{N_{H}}\right\}$ are the quadratic $P_{2}$ local basis functions in the host element $\widetilde{\mathcal{T}}_{j}$. As stated above, high-order Lagrange interpolations yield numerical solutions 
polluted with non-physical oscillations and do not satisfy the discrete maximum principle.

4. Calculate the linear $P_{1}$ finite element approximation

$$
\widetilde{u}_{L j}^{n}=\sum_{k=1}^{N_{L}} \widetilde{U}_{k}^{n} \psi_{k}\left(\boldsymbol{X}_{h j}^{n}\right),
$$

where $\left\{\psi_{1}, \cdots, \psi_{N_{L}}\right\}$ are the linear $P_{1}$ local basis functions on the element $\widetilde{\mathcal{T}}_{j}$. Recall that the linear Lagrange interpolation is monotone and the numerical solutions obtained using the linear $P_{1}$ interpolation are free of non-physical oscillations and artificial extrema.

5. Calculate the limited solution $\widetilde{u}_{j}^{n}$ using a convex combination of the quadratic $P_{2}$ solution (3.1) and the linear $P_{1}$ solution (3.2) as

$$
\widetilde{u}_{j}^{n}=\widetilde{u}_{L j}^{n}+\alpha_{j}^{n}\left(\widetilde{u}_{H j}^{n}-\widetilde{u}_{L j}^{n}\right),
$$

where $\alpha_{j}^{n} \in[0,1]$ is a limiting function used to adjust the amount of correction in the linear $P_{1}$ approximation in order to obtain a non-oscillatory solution. It is evident that for $\alpha_{j}^{n}=0$, the obtained solution in (3.3) reduces to the linear approximation, whereas the quadratic $P_{2}$ approximation is recovered for $\alpha_{j}^{n}=1$. In the present work, the limiting function $\alpha_{j}^{n}$ is locally chosen such that the approximate finite element solution (3.3) is monotone i.e. the solution $\widetilde{u}_{j}^{n}$ in (3.3) remains bounded in $\left[u_{j}^{-}, u_{j}^{+}\right]$ at each time step, where $u_{j}^{+}$and $u_{j}^{-}$are respectively, the maximum and minimum of the nodal solutions in the host element $\widetilde{\mathcal{T}}_{j}$ defined as

$$
u_{j}^{-}=\min \left(\widetilde{U}_{1}^{n}, \cdots, \widetilde{U}_{N_{H}}^{n}\right), \quad u_{j}^{+}=\max \left(\widetilde{U}_{1}^{n}, \cdots, \widetilde{U}_{N_{H}}^{n}\right) .
$$

To improve the accuracy and to minimize the numerical dissipation in the linear $P_{1}$ finite element approximation, we propose a local limiting function $\alpha_{j}^{n}$ defined using the slope of the solution as

$$
\alpha_{j}^{n}= \begin{cases}\min \left(1, \frac{u_{j}^{+}-\widetilde{u}_{L j}^{n}}{\left.\widetilde{u}_{L j}^{n}-\widetilde{u}_{H j}^{n}\right),}\right. & \text { if } \widetilde{u}_{L j}^{n}-\widetilde{u}_{H j}^{n}>0, \\ \min \left(1, \frac{u_{j}^{-}-\widetilde{u}_{L j}^{n}}{\widetilde{u}_{L j}^{n}-\widetilde{u}_{H j}^{n}}\right), & \text { if } \widetilde{u}_{L j}^{n}-\widetilde{u}_{H j}^{n}<0, \\ 1, & \text { if } \widetilde{u}_{L j}^{n}-\widetilde{u}_{H j}^{n}=0 .\end{cases}
$$


6. In general the above limiting procedure does not conserve the mass i.e.

$$
\int_{\Omega} \widetilde{u}^{n}(x) d x \neq \int_{\Omega} u_{0}(x) d x
$$

and therefore, the solution $\widetilde{u}_{j}^{n}$ in (3.3) should be corrected to be fully conservative. To achieve this property in our characteristic finite element method we correct the computed solution by the difference between the linear $P_{1}$ and quadratic $P_{2}$ solutions multiplied by a suitable coefficient as follows

$$
u_{j}^{n+1}=\widetilde{u}_{j}^{n}+\theta \max \left(0, \operatorname{sign}(\Delta \mathcal{M})\left(\widetilde{u}_{H j}^{n}-\widetilde{u}_{L j}^{n}\right)^{3}\right)
$$

where $\theta$ and $\Delta \mathcal{M}$ are computed at each time step $t_{n}$ as

- Calculate the mass difference between the initial solution and the limited solution as

$$
\Delta \mathcal{M}=\int_{\Omega} \widetilde{u}^{n}(\boldsymbol{x}) d x-\int_{\Omega} u_{0}(\boldsymbol{x}) d \boldsymbol{x} .
$$

- Check if $\Delta \mathcal{M}=0$ then the computed solution satisfies the mass conservation propriety. If not we compute the correction coefficient $\omega_{j}$ as

$$
\omega_{j}=\max \left(0, \operatorname{sign}(\Delta \mathcal{M})\left(\widetilde{u}_{H j}^{n}-\widetilde{u}_{L j}^{n}\right)^{3}\right) .
$$

- Compute the correction $\theta$ as

$$
\theta=\frac{\Delta \mathcal{M}}{\int_{\Omega} \omega_{h}(x) d x},
$$

where $\omega_{h}(x)=\sum_{j=1}^{M} \omega_{j} \phi_{H j}(x)$.

It is worth remarking that this procedure evaluate the nodal values of the numerical solution by adding to the monotone linear $P_{1}$ solution a correction accounting for the quadratic $P_{2}$ solution without violating the monotonicity of the linear $P_{1}$ solution. In fact, using the limiting approach (3.3)-(3.5), the obtained finite element solution is conservative and it remains within the largest and the smallest values of the solution in a set of nodal points surrounding the departure point $\boldsymbol{X}_{h j}^{n}$. As a consequence, the reconstructed nodal solution (3.3) does not generate any extrema which does not exists in the solutions at the neighborhood of the departure points. Note that for the mesh elements illustrated in Fig. 1, the numbers of linear $P_{1}$ and quadratic $P_{2}$ local basis functions are $N_{L}=4$ and $N_{H}=10$, respectively. 


\section{Application to incompressible Navier-Stokes equations}

In this section, we extend the characteristic finite element method for the incompressible Navier-Stokes equations reformulated in dimensionless primitive variables as

$$
\begin{aligned}
& \nabla \cdot \boldsymbol{u}=0, \\
& \frac{\partial \boldsymbol{u}}{\partial t}+\boldsymbol{u} \cdot \nabla \boldsymbol{u}+\nabla p-\frac{1}{R e} \Delta \boldsymbol{u}=\mathbf{f},
\end{aligned}
$$

where $p$ is the pressure, $\boldsymbol{u}=(u, v, w)^{\top}$ the velocity field, with $u$ the velocity in $x$-direction, $v$ the velocity in $y$-direction, $w$ the velocity in $z$-direction, $\mathbf{f}$ the external force, and $R e$ the Reynolds number. Recall that this non-dimensional number is usually used to control the relative dominance of the convection compared to the diffusion in (4.1). For a well-posed mathematical problem, Eq. (4.1) are solved in a three-dimensional bounded domain $\Omega \subset$ $\mathbb{R}^{3}$ with Lipschitz boundary $\Gamma$ subject to well-defined boundary and initial conditions.

In general, the characteristic finite element method belongs to fractional step techniques where the transport part in (4.1) is decoupled from the Stokes part in the time integration. Thus, at each time step the velocity and pressure are updated by solving first the transport equation

$$
\frac{D \boldsymbol{u}}{D t}:=\frac{\partial \boldsymbol{u}}{\partial t}+\boldsymbol{u} \cdot \nabla \boldsymbol{u}=\mathbf{0},
$$

followed by the Stokes equations

$$
\begin{aligned}
& \nabla \cdot \boldsymbol{u}=0 \\
& \frac{D \boldsymbol{u}}{D t}+\nabla p-\frac{1}{R \boldsymbol{e}} \Delta \boldsymbol{u}=\mathbf{f} .
\end{aligned}
$$

In this study, the conforming finite element spaces for the pressure and velocity solutions are the mixed Taylor-Hood finite elements $P_{1}-P_{2}$ illustrated in Fig. 1 . Here, the linear $P_{1}$ finite elements are used for the pressure and the quadratic $P_{2}$ finite elements are used for the velocity. Notice that for these mixed finite elements, the discrete velocity and pressure solutions satisfy the well-established inf-sup condition, see for instance [18]. The associated finite element spaces are defined as

$$
\begin{aligned}
& \mathbf{V}_{h}=\left\{\boldsymbol{u}_{h} \in C^{0}(\bar{\Omega}) \times C^{0}(\bar{\Omega}) \times C^{0}(\bar{\Omega}):\left.\boldsymbol{u}_{h}\right|_{\mathcal{T}_{k}} \in P_{2}\left(\mathcal{T}_{k}\right), \forall \mathcal{T}_{k} \in \Omega_{h}\right\}, \\
& Q_{h}=\left\{p_{h} \in C^{0}(\bar{\Omega}):\left.p_{h}\right|_{\mathcal{T}_{k}} \in P_{1}\left(\mathcal{T}_{k}\right), \forall \mathcal{T}_{k} \in \Omega_{h}\right\},
\end{aligned}
$$

where $P_{1}\left(\mathcal{T}_{j}\right)$ and $P_{2}\left(\mathcal{T}_{j}\right)$ are polynomial spaces defined in the finite element $\mathcal{T}_{k}$. Hence, we approximate the finite element solutions to $\boldsymbol{u}^{n}(\boldsymbol{x})$ and $p^{n}(\boldsymbol{x})$ as

$$
p_{h}^{n}(\boldsymbol{x})=\sum_{l=1}^{M_{p}} P_{l}^{n} \psi_{l}(\boldsymbol{x}), \quad \boldsymbol{u}_{h}^{n}(\boldsymbol{x})=\sum_{j=1}^{M} \boldsymbol{U}_{j}^{n} \phi_{j}(\boldsymbol{x}),
$$


where $M_{p}$ and $M$ are respectively, the number of pressure and velocity nodal points in $\Omega_{h}$. The functions $P_{l}^{n}$ and $\boldsymbol{U}_{j}^{n}=\left(U_{j}^{n}, V_{j}^{n}, W_{j}^{n}\right)^{\top}$ are the corresponding nodal values of $p_{h}^{n}(\boldsymbol{x})$ and $\boldsymbol{u}_{h}^{n}(\boldsymbol{x})$, respectively. These functions satisfy $P_{l}^{n}=p_{h}^{n}\left(\boldsymbol{y}_{l}\right)$ and $\boldsymbol{U}_{j}^{n}=\boldsymbol{u}_{h}^{n}\left(\boldsymbol{x}_{j}\right)$, with $\left\{\boldsymbol{y}_{l}\right\}_{l=1}^{M_{p}}$ and $\left\{\boldsymbol{x}_{j}\right\}_{j=1}^{M}$ are the set of pressure and velocity mesh points in $\Omega_{h}$, respectively, so that $M_{p}<M$ and $\left\{\boldsymbol{y}_{1}, \cdots, \boldsymbol{y}_{M_{p}}\right\} \subset\left\{\boldsymbol{x}_{1}, \cdots, \boldsymbol{x}_{M}\right\}$. Here, $\left\{\psi_{l}\right\}_{l=1}^{M_{p}}$ and $\left\{\phi_{j}\right\}_{j=1}^{M}$ are respectively, the set of global nodal basis functions of the pressure and the velocity characterized by the property $\phi_{i}\left(\boldsymbol{x}_{j}\right)=\delta_{i j}$ and $\psi_{i}\left(\boldsymbol{y}_{l}\right)=\delta_{i l}$ with $\delta$ denoting the Kronecker symbol.

To solve the Stokes problem (4.3) in the current work, we implement an efficient direct-type algorithm, see $[18,23]$ for similar solvers. The main advantage of this algorithm is the fact that neither projection techniques nor special corrections for the pressure are required for the solution of the Stokes problem. Unlike the first-order implicit Euler scheme used for the time integration in $[18,23]$, we consider the second-order implicit BDF2 scheme in the present study. Thus, given a tolerance $\varepsilon$ and using superscripts in parenthesis to denote the iteration numbers, the procedure to advance the solution of (4.3) from the current time $t_{n}$ to the next time $t_{n+1}$ is carried out using the following steps:

1. Given $p_{h}^{(0)}=p_{h}^{n}$, solve for $\boldsymbol{u}_{h}^{(0)} \in \mathbf{V}_{h}$ such that for all $\boldsymbol{v}_{h} \in \mathbf{V}_{h}$

$$
\begin{aligned}
\frac{3}{2 \Delta t} \int_{\Omega} \boldsymbol{u}_{h}^{(0)} \boldsymbol{v}_{h} d \boldsymbol{x}+\frac{1}{R e} \int_{\Omega} \nabla \boldsymbol{u}_{h}^{(0)} \cdot \nabla \boldsymbol{v}_{h} d \boldsymbol{x}= & \int_{\Omega} p_{h}^{(0)} \nabla \cdot \boldsymbol{v}_{h} d \boldsymbol{x}+\int_{\Omega} \mathbf{f}_{h}^{n+1} \boldsymbol{v}_{h} d \boldsymbol{x} \\
& +\frac{1}{2 \Delta t} \int_{\Omega}\left(4 \widetilde{\boldsymbol{u}}_{h}^{n}-\widetilde{\boldsymbol{u}}_{h}^{n-1}\right) \boldsymbol{v}_{h} d \boldsymbol{x} .
\end{aligned}
$$

Then, compute

$$
q_{h}^{(0)}=\nabla \cdot \boldsymbol{u}_{h}^{(0)}
$$

2. Solve for $\psi_{h}^{(0)} \in Q_{h}$ such that for all $\phi_{h} \in Q_{h}$

$$
\int_{\Omega} \nabla \psi_{h}^{(0)} \cdot \nabla \phi_{h} d x=\int_{\Omega} q_{h}^{(0)} \phi_{h} d x
$$

and set

$$
\zeta_{h}^{(0)}=\frac{3}{2 \Delta t} \psi_{h}^{(0)}+\frac{1}{R e} q_{h}^{(0)}, \quad \xi_{h}^{(0)}=\zeta_{h}^{(0)}
$$

3. For $m=1,2, \cdots$, assume that $p_{h}^{(m)}, \boldsymbol{u}_{h}^{(m)}, q_{h}^{(m)}, \zeta_{h}^{(m)}, \xi_{h}^{(m)}$ are known, we compute $p_{h}^{(m+1)}, \boldsymbol{u}_{h}^{(m+1)}, q_{h}^{(m+1)}, \zeta_{h}^{(m+1)}, \xi_{h}^{(m+1)}$ as follows:

(a) Solve for $\overline{\boldsymbol{u}}_{h} \in \mathbf{V}_{h}$ such that for all $\boldsymbol{v}_{h} \in \mathbf{V}_{h}$

$$
\frac{3}{2 \Delta t} \int \overline{\boldsymbol{u}}_{h}^{(m)} \boldsymbol{v}_{h} d \boldsymbol{x}+\frac{1}{\operatorname{Re}} \int \nabla \overline{\boldsymbol{u}}_{h}^{(m)} \cdot \nabla \boldsymbol{v}_{h} d \boldsymbol{x}=\int \xi_{h}^{(m)} \nabla \cdot \boldsymbol{v}_{h} d \boldsymbol{x},
$$

and set

$$
\bar{q}_{h}^{(m)}=\nabla \cdot \overline{\boldsymbol{u}}_{h}^{(m)}
$$


(b) Compute

$$
\eta_{m}=\frac{\int q_{h}^{(m)} \zeta_{h}^{(m)} d x}{\int \bar{q}_{h}^{(m)} \xi_{h}^{(m)} d x}
$$

(c) Set

$$
p_{h}^{(m+1)}=p_{h}^{(m)}-\eta_{m} \xi_{h}^{(m)}, \quad \boldsymbol{u}_{h}^{(m+1)}=\boldsymbol{u}_{h}^{(m)}-\eta_{m} \overline{\boldsymbol{u}}_{h}^{(m)}, \quad q_{h}^{(m+1)}=q_{h}^{(m)}-\eta_{m} \bar{q}_{h}^{(m)} .
$$

(d) Solve for $\bar{\psi}_{h}^{(m)} \in Q_{h}$ such that for all $\phi_{h} \in Q_{h}$

$$
\int \nabla \bar{\psi}_{h}^{(m)} \cdot \nabla \phi_{h} d x=\int \bar{q}_{h}^{(m)} \phi_{h} d x
$$

and set

$$
\zeta_{h}^{(m+1)}=\zeta_{h}^{(m)}-\eta_{m}\left(\frac{3}{2 \Delta t} \bar{\psi}_{h}^{(m)}+\frac{1}{R e} q_{h}^{(m)}\right)
$$

i. If

$$
\frac{\int q_{h}^{(m+1)} \zeta_{h}^{(m+1)} d x}{\int q_{h}^{(0)} \zeta_{h}^{(0)} d x} \leq \varepsilon
$$

then

$$
p_{h}^{n+1}=p_{h}^{(m+1)}, \quad \boldsymbol{u}_{h}^{n+1}=\boldsymbol{u}_{h}^{(m+1)},
$$

stop.

ii. Else, compute

$$
\chi_{m}=\frac{\int q_{h}^{(m+1)} \zeta_{h}^{(m+1)} d x}{\int q_{h}^{(m)} \zeta_{h}^{(m)} d x}, \quad \xi_{h}^{(m+1)}=\zeta_{h}^{(m+1)}+\chi_{m} \xi_{h}^{(m)},
$$

change $m \longleftarrow m+1$, return to step (a) and repeat.

iii. End if

It is evident that the iterative procedure in the above direct-type algorithm involves solutions of uncoupled elliptic problems such that their finite element discretization yields well-conditioned linear systems of algebraic equations for which very efficient iterative solvers can be implemented. In our computations, taking advantage of these properties we solve these linear systems in the characteristic finite element method using the conjugate gradient solver using an incomplete Cholesky factorization. Note that the finite element discretization of Eqs. (4.5)-(4.8) is trivial and for brevity in the presentation it is omitted here. 


\section{Numerical results}

We examine the accuracy of the new conservative and monotone characteristic finite element method introduced in the above sections using several numerical examples for convection-dominated problems. For the example with known analytical solution we compute the total error as

$$
E_{T o t}=\int_{\Omega}\left(u-u_{\text {exact }}\right)^{2} d x,
$$

where $u$ and $u_{\text {exact }}$ are the numerical solution and the exact solution, respectively. We also evaluate the dissipation error $E_{D i s s}$ and the dispersion error $E_{D i s p}$ as

$$
E_{\text {Diss }}=\left(\sigma(u)-\sigma\left(u_{\text {exact }}\right)\right)^{2}+\left(\bar{u}-\bar{u}_{\text {exact }}\right)^{2}, \quad E_{\text {Disp }}=2(1-\rho) \sigma(u) \sigma\left(u_{\text {exact }}\right),
$$

where $\bar{u}$ and $\sigma(u)$ are respectively, the mean and standard deviation of the solution $u$, and $\rho$ is the correlation coefficient between $u$ and $u_{\text {exact }}$. Note that as shown in [42]

$$
E_{T o t}=E_{\text {Diss }}+E_{\text {Disp }}
$$

We also define the CFL number as

$$
\mathrm{CFL}=\max _{x, y, z}\left(\sqrt{v_{1}^{2}+v_{2}^{2}+v_{3}^{2}}\right) \frac{\Delta t}{h} .
$$

In our simulations presented in this section, the CFL number is set to a fixed value and the time stepsize $\Delta t$ is adjusted at each time step according to the condition (5.2). To solve the resulting linear systems of algebraic equations, we use the preconditioned conjugate gradient algorithm equipped with a tolerance of $10^{-7}$ to stop the iterations. Note that to minimize the computational effort in the proposed characteristic finite element method, values of the CFL number are selected as large as possible for which the explicit Eulerianbased finite element methods are noncompetitive for three-dimensional problems. In this study, we also compare numerical results obtained using the linear $P_{1}$ elements, the quadratic $P_{2}$ elements and the proposed mass-conservative and monotonicity-preserving procedure. All the simulations reported in this section were performed on a Pentium PC with Intel ${ }^{\circledR}$ Core i7-7700HQ of 8 GB of RAM and $8 \mathrm{GHz}$ using serial Fortran compiler.

\subsection{Rotating a slotted sphere in circular flow fields}

We consider the problem of a slotted sphere in circular flow fields widely used in the literature to assess the numerical performance of transport methods for problems with sharp discontinuities. Note that this problem is an extension to three space dimensions of the well known two-dimensional Zalezak's slotted disk proposed in [47]. The problem statement consists on solving Eq. (2.1) in the spatial domain $\Omega=[-0.5,0.5] \times[-0.5,0.5] \times$ $[-0.5,0.5]$ equipped with the flow field $v=(-\omega y, \omega x, 0)^{\top}$, where $\omega=4$ is the angular velocity. The solution is a sphere centered at $(-0.25,0,0)$ of radius 0.15 and height of 

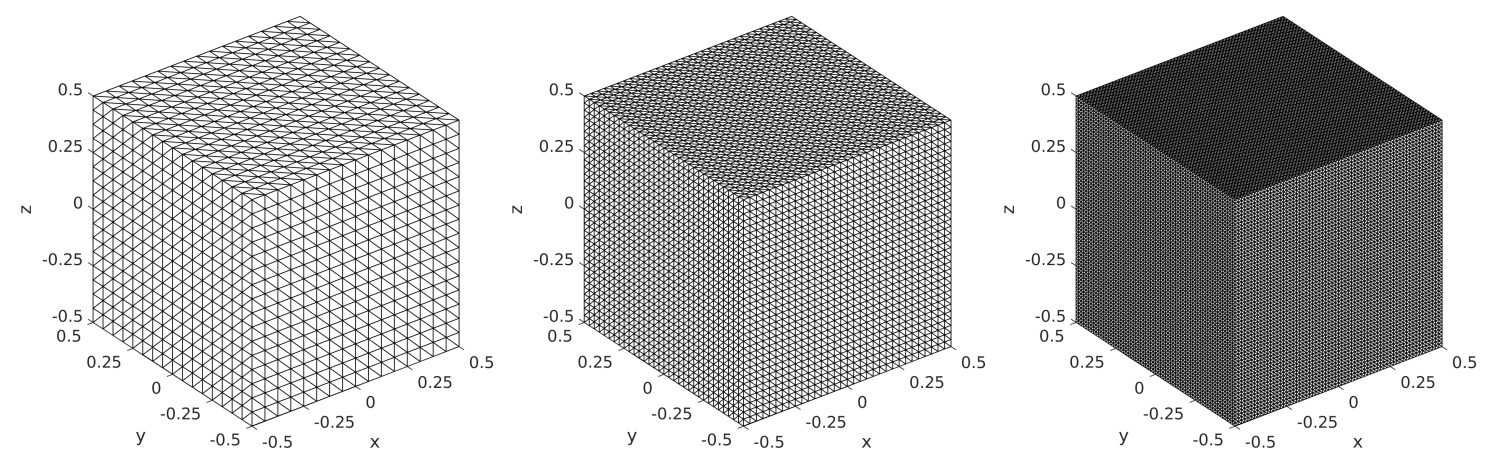

Figure 2: Uniform finite element meshes with spatial steps $h=\frac{1}{32}$ (left), $h=\frac{1}{64}$ (middle) and $h=\frac{1}{128}$ (right) used for transport problems in rotating a slotted sphere in circular flow fields.
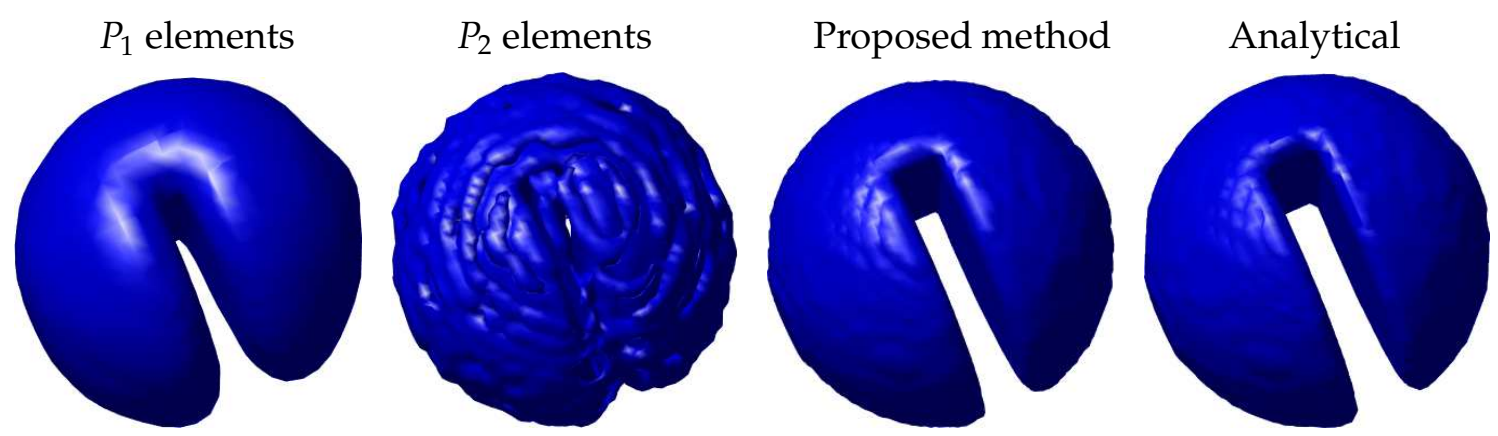

Figure 3: Iso-surfaces for rotating a slotted sphere in circular flow fields using $\mathrm{CFL}=10$ and $h=\frac{1}{128}$.

1 along with a slot in the $x y$-plane of width 0.06 and a length of 0.22 . In absence of diffusion in the problem, the slotted sphere is expected to preserve its shape and mass during the time integration and the total time required for one complete rotation is $\frac{\pi}{2}$. In our simulations for this example, we consider three uniform meshes with $h=\frac{1}{32}, h=\frac{1}{64}$ and $h=\frac{1}{128}$ as shown in Fig. 2, and three different values for CFL namely CFL $=2.5,5$ and 10.

In Fig. 3 we present the plots for iso-surfaces of the computed solutions after one rotation using the mesh with $h=\frac{1}{128}$ and CFL $=10$. We include the analytical solution and the numerical solutions obtained using the linear $P_{1}$ elements, the quadratic $P_{2}$ elements and the proposed conservative and monotonicity-preserving method. As can be seen from these results, the proposed method preserves the shape of the numerical solution with very little numerical diffusion compared to the solutions computed using the $P_{2}$ elements and the $P_{1}$ elements. Compared to the exact solution, the numerical solution obtained using the linear $P_{1}$ elements exhibits large numerical diffusion whereas, the non-physical solutions are very remarkable in the numerical solution obtained using the quadratic $P_{2}$ elements. For a better visualization, Fig. 4 illustrates contourlines in the $x y$-plane at $z=0$ of the exact solution and the numerical solutions obtained using the linear $P_{1}$ elements, 

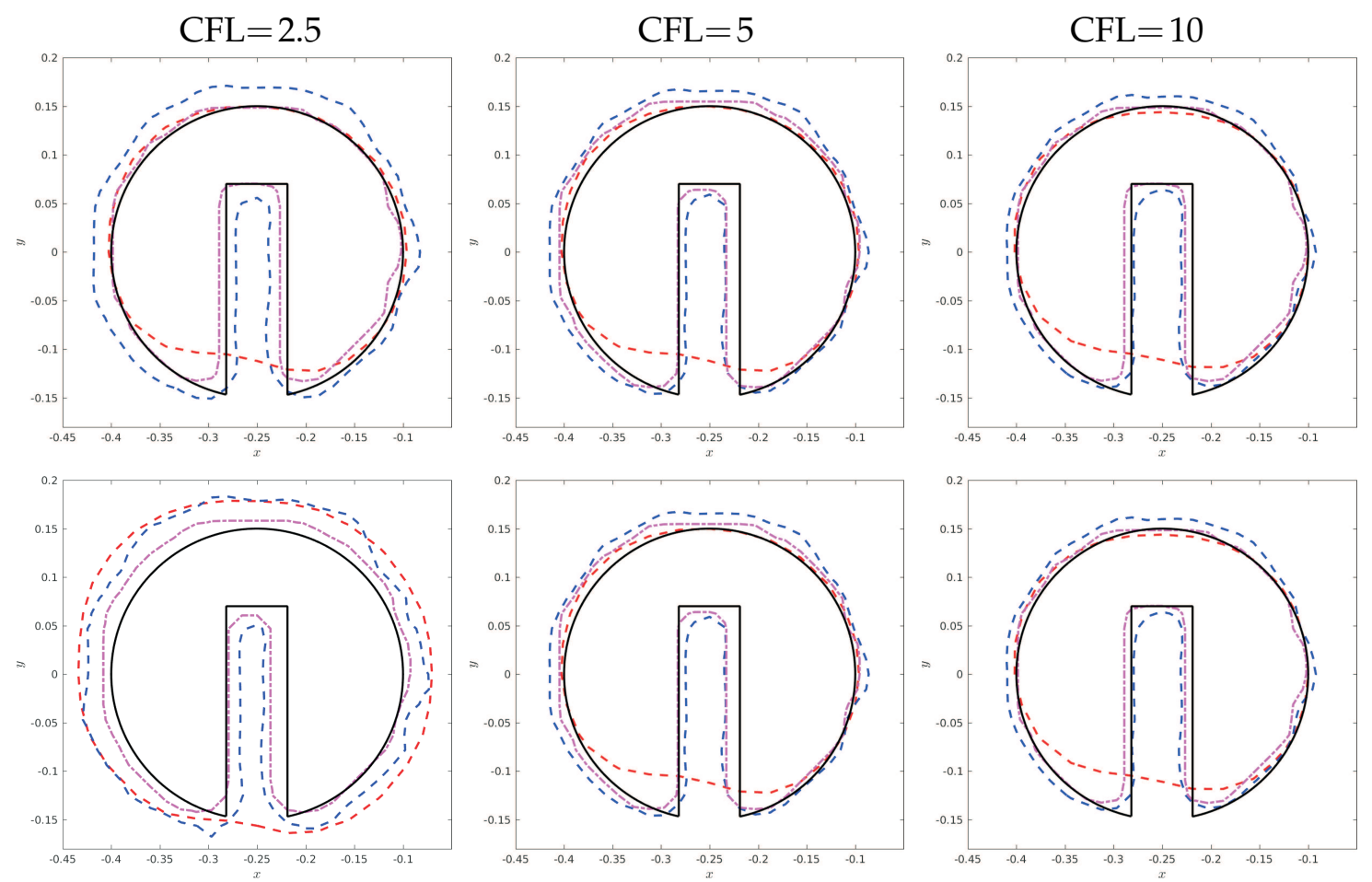

$-\ldots \mathrm{P}_{1}$ Elements - .-- $\mathrm{P}_{2}$ Elements

Proposed method

Analytical

Figure 4: Contourlines of the solutions at $z=0$ for rotating a slotted sphere in circular flow fields after one revolution (first row) and after two revolutions (second row) using $h=\frac{1}{64}$ and different CFL numbers.

the quadratic $P_{2}$ elements and the proposed conservative and monotonicity-preserving method after one and two revolutions on the mesh with $h=\frac{1}{64}$ and different CFL numbers. Note that only part of the computational domain is shown in these plots for a better insight. To further compare these results, we show in Fig. 5 and Fig. 6 the one-dimensional cross-sections along the horizontal line at $y=z=0$ for the obtained solutions using three different values of $\mathrm{CFL}=2.5,5$ and 10 after one and two rotations. A visual comparison of the obtained results demonstrates large numerical dissipation, severe overshoots, deformation and phase errors in the numerical solutions computed using the linear $P_{1}$ and quadratic $P_{2}$ elements. After one rotation, the results obtained using the quadratic $P_{2}$ elements exhibit non-physical oscillations and greater distortions localized mainly at the feet and the upper face of the slotted sphere where discontinuities are more sharper than elsewhere in the computed solutions. After two rotations, the magnitude and frequency of these non-physical oscillations increase in the computed solutions using the quadratic $P_{2}$ elements. Contrarily, numerical dissipation in the results obtained using the linear $P_{1}$ elements is clearly noticeable and it becomes more pronounced for small values of CFL. As expected, refining the finite element mesh results in an increase in the accuracy of the 

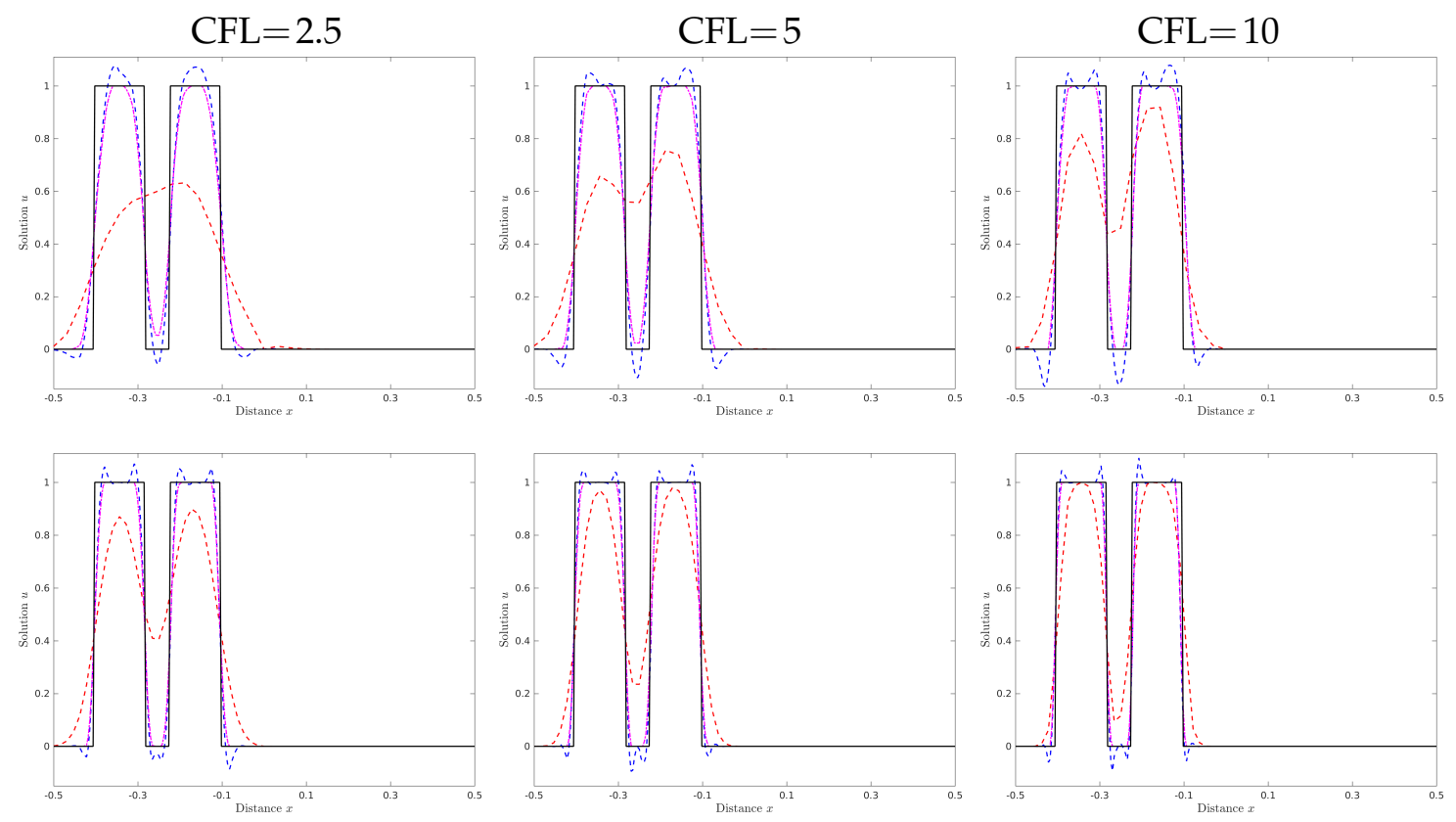

- - - $\mathrm{P}_{1}$ Elements _- - $\mathrm{P}_{2}$ Elements _...... Proposed method

Analytical

Figure 5: Cross-sections of the solution at $y=z=0$ for rotating a slotted sphere in circular flow fields after 1 revolution using different CFL and meshes with $h=\frac{1}{64}$ (first row) and $h=\frac{1}{128}$ (second row).

computed solutions. For instance, in a mesh with $h=\frac{1}{64}$ and after two rotations, the solutions obtained using the linear $P_{1}$ elements demonstrate substantially large numerical diffusion at the feet of the slotted sphere where gradients are sharp, compare the results shown in Fig. 4 and Fig. 6. However, from the same figures we observe a full absence of these numerical dissipation and non-physical oscillations in the results obtained using our conservative and monotonicity-preserving method. It should also be stressed that the numerical accuracy in the proposed characteristic finite element method increases for large value of CFL numbers, compare the results obtained using the low $\mathrm{CFL}=2.5$ and the high $\mathrm{CFL}=10$ in Fig. 4 and Fig. 5. It is also evident that the proposed conservative and monotonicity-preserving procedure eliminates the non-physical oscillations in the vicinity of feet of the sphere where discontinuities are sharp and the transport is well resolved without requiring fine meshes or small time steps.

Next we perform quantitative comparisons of the results obtained using the linear $P_{1}$ elements, the quadratic $P_{2}$ elements and the proposed conservative and monotonicitypreserving method. In Table 1 we summarize the obtained results after one and two rotations using different values of CFL and mesh densities. We report the minimum (Min) and the maximum (Max) values of the computed solutions, relative mass (Mass), the errors $E_{\text {Diss, }}, E_{\text {Disp }}$ and $E_{\text {Tot }}$ and the computational times (CPU) given in seconds. 


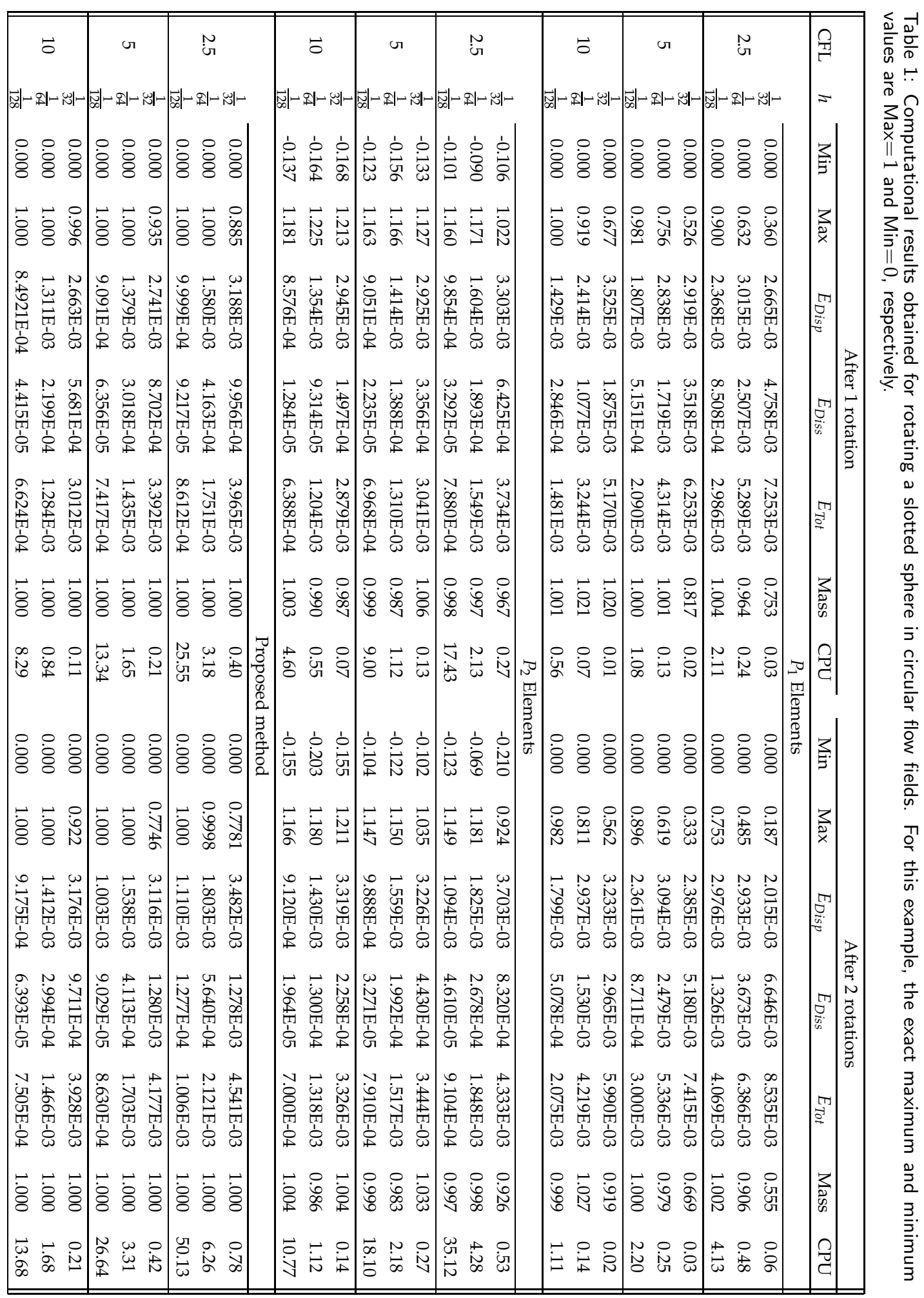



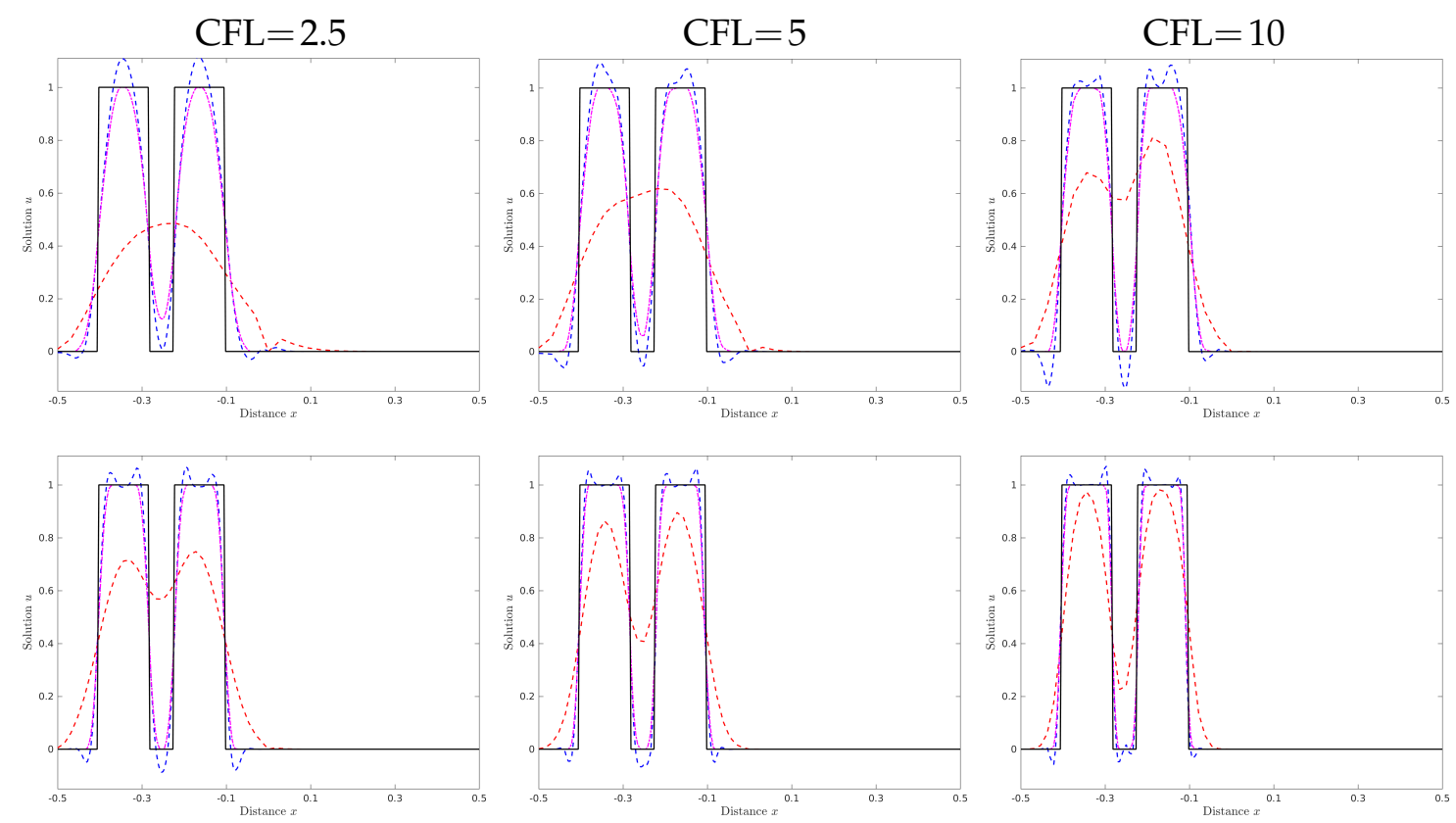

- - - $P_{1}$ Elements _ - - $P_{2}$ Elements _...... Proposed method

Analytical

Figure 6: Same as Fig. 5 but after two rotations.

For the meshes and numbers of revolutions considered, the proposed conservative and monotonicity-preserving procedure conserves the mass at a minor additional effort referring to the CPU time. For the considered transport conditions, failure of mass conservation is clear in the results obtained using the linear $P_{1}$ and quadratic $P_{2}$ elements whereas, the relative mass remains fixed to unity in the proposed conservative characteristic finite element method. In terms of the considered errors $E_{\text {Diss }}, E_{D i s p}$ and $E_{T o t}$, the results obtained using the quadratic $P_{2}$ elements are more accurate than those obtained using the linear $P_{1}$ elements for all the considered meshes and CFL numbers. It is also evident that the proposed conservative and monotonicity-preserving procedure does not deteriorate the overall accuracy of the characteristic finite element method. In addition, increasing the CFL number results in a decrease of the total error in all computed solutions. However, only results obtained using the proposed method yield a reduction of the dispersion error $E_{\text {Disp }}$ as CFL increases. Furthermore, results obtained in Table 1 for the errors $E_{\text {Diss, }}$ $E_{D i s p}$ and $E_{\text {Tot }}$ using the proposed conservative and monotonicity-preserving method confirm that these results are monotone and free from non-physical oscillations during the time integration process. Indeed, the conservative and monotonicity-preserving method captures the physics well in this three-dimensional transport problem. From the values of Max and Min listed in Table 1, we observe very low values of Max for the results obtained using the linear $P_{1}$ elements and high and negative values for the results obtained using the quadratic $P_{2}$ elements which are removed in the results obtained 
using the conservative and monotonicity-preserving procedure. It is also clear that the CPU times in the characteristic finite element method using the quadratic $P_{2}$ elements are higher than the CPU times using the linear $P_{1}$ elements. For the considered transport conditions, the CPU time of the quadratic $P_{2}$ elements is about 9 times larger than the CPU time of the linear $P_{1}$ elements. However, the difference between the CPU times for the quadratic $P_{2}$ elements and conservative and monotonicity-preserving method it minimal and it is about $15 \%$ for all the simulations. It should also be noted that the characteristic finite element method is commonly designed to solve this class of transport problems using CFL numbers four to five times larger than its Eulerian counterparts.

\subsection{A deformational flow problem}

In this example we solve a deformational flow problem widely used in $[30,40]$ to examine the performance of high-resolution conservative algorithms for advection problems using structured meshes. In the present study we solve this problem in a sphere using unstructured tetrahedral meshes. The flow field is reconstructed in this example by superimposing both deformation in the $x y$-plane with deformation in the $x z$-plane. Thus, we solve the transport equation (2.1) in a sphere centered at $(0,0,0)^{\top}$, with radius 0.6 subject to a velocity field defined by

$$
\begin{aligned}
& v_{1}(x, y, z, t)=2 \sin ^{2}(\pi x) \sin (\pi y) \sin (\pi z) \cos \left(\frac{\pi t}{T}\right), \\
& v_{2}(x, y, z, t)=-\sin (\pi x) \sin ^{2}(\pi y) \sin (\pi z) \cos \left(\frac{\pi t}{T}\right), \\
& v_{3}(x, y, z, t)=-\sin (\pi x) \sin (\pi y) \sin ^{2}(\pi z) \cos \left(\frac{\pi t}{T}\right),
\end{aligned}
$$

where $T$ is the final time period. As stated in $[30,40]$, the flow becomes slow and it changes the direction in such a way that the initial condition is recovered at time $T$, i.e. $u(x, y, z, 0)=u(x, y, z, T)$. Therefore, this flow problem is very interesting since the analytical solution at the final time $T$ is known even though the flow structure becomes complicated at this time. In our simulations, the time period $T=1.5$ and initially

$$
u(x, y, z, 0)= \begin{cases}1, & \text { if } x \leq \frac{1}{2} \\ 0, & \text { if } x>\frac{1}{2}\end{cases}
$$

Note that for this initial condition, the interface at $x=\frac{1}{2}$ deforms in a fully threedimensional manner and returns to its initial location at the final time $t=T$. For the results presented for this example, we use CFL $=10$ and three unstructured meshes as shown in Fig. 7. The corresponding statistics of elements and nodal points for these meshes are summarized in Table 2. 

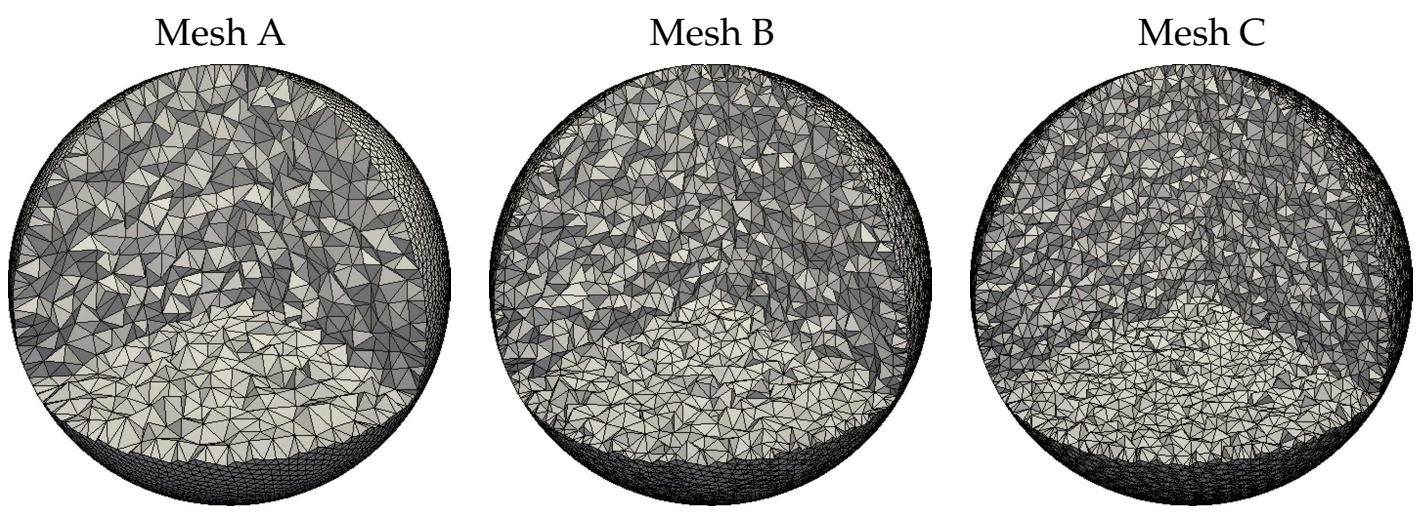

Figure 7: Unstructured finite element meshes with 36844 elements (left), 52943 elements (middle) and 119604 elements (right) used for the deformational flow problem.

Table 2: Numbers of elements and nodes in the meshes used for the deformational flow problem.

\begin{tabular}{||cccc||}
\hline Mesh & \# of elements & \# of $P_{2}$ nodes & \# of $P_{1}$ nodes \\
\hline Mesh A & 36844 & 64003 & 9977 \\
Mesh B & 52943 & 84934 & 12388 \\
Mesh C & 119604 & 183495 & 25563 \\
\hline
\end{tabular}

In Fig. 8 we display the results obtained using the quadratic $P_{2}$ elements and the proposed conservative and monotone method on the three considered meshes at time $t=\frac{T}{2}$. Those results obtained at time $t=T$ are presented in Fig. 9. For better insight, only half of the sphere is illustrated for the computed solutions in these figures. At time $t=\frac{T}{2}$, the interface at the sphere center appears disconnected whereas at the final time $t=T$ the initial interface is recovered subject to a non-preventable smearing generated by the full three-dimensional deformational flow field. It is also clear from the results in Fig. 8 and Fig. 9 that the results obtained using the quadratic $P_{2}$ elements exhibit oscillatory behavior at both times especially at the vicinity of sharp gradients in the computational domain on coarse meshes Mesh A and Mesh B. Furthermore, non-physical negative values are clearly detected in results obtained using the quadratic $P_{2}$ elements. Refining the mesh results in an improvement in the accuracy for all results. From the same figures, the results obtained using the proposed conservative method are free from non-physical negative values and capture the correct interface on the three considered meshes and at both times $t=\frac{T}{2}$ and $t=T$. Note that the results shown here agree well with those presented in $[30,40]$ for the cuboid domain. It should be pointed out that the numerical methods in $[30,40]$ used a high resolution scheme based on upwind techniques for which direct or approximate Riemann solvers are needed. In contrast, our characteristic finite element method does not require any Riemann solver and it produces stable results which are conservative, monotone and comparable to those obtained by upwinding in [30,40]. For comparison reasons, Fig. 10 illustrates the solution cross-sections along the radial line at 

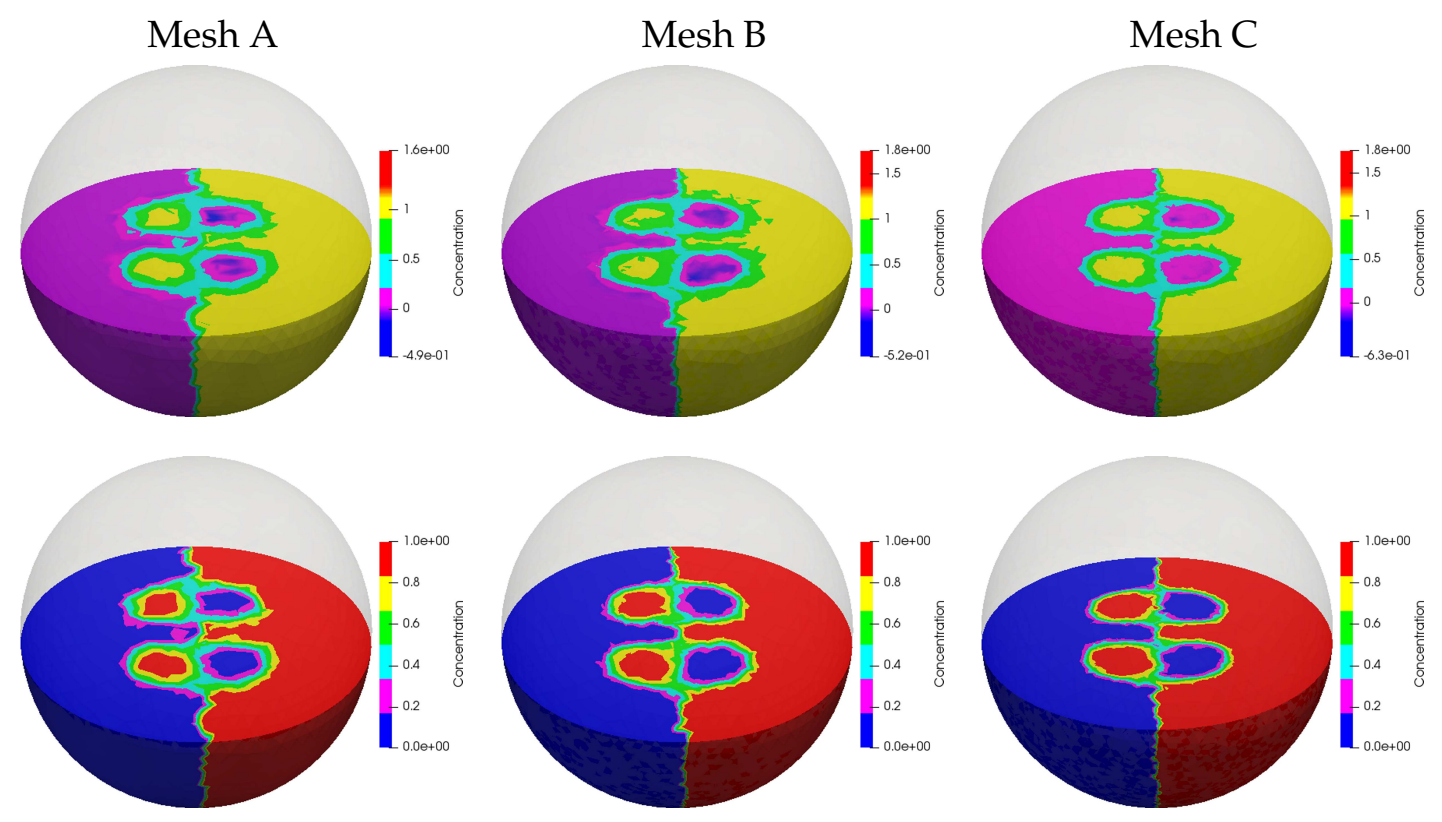

Figure 8: Numerical results obtained for the deformational flow problem on Mesh A (first column), Mesh B (second column) and Mesh C (third column) using the $P_{2}$ elements (first row) and the proposed conservative and monotonicity-preserving method (second row) at time $t=\frac{T}{2}$.
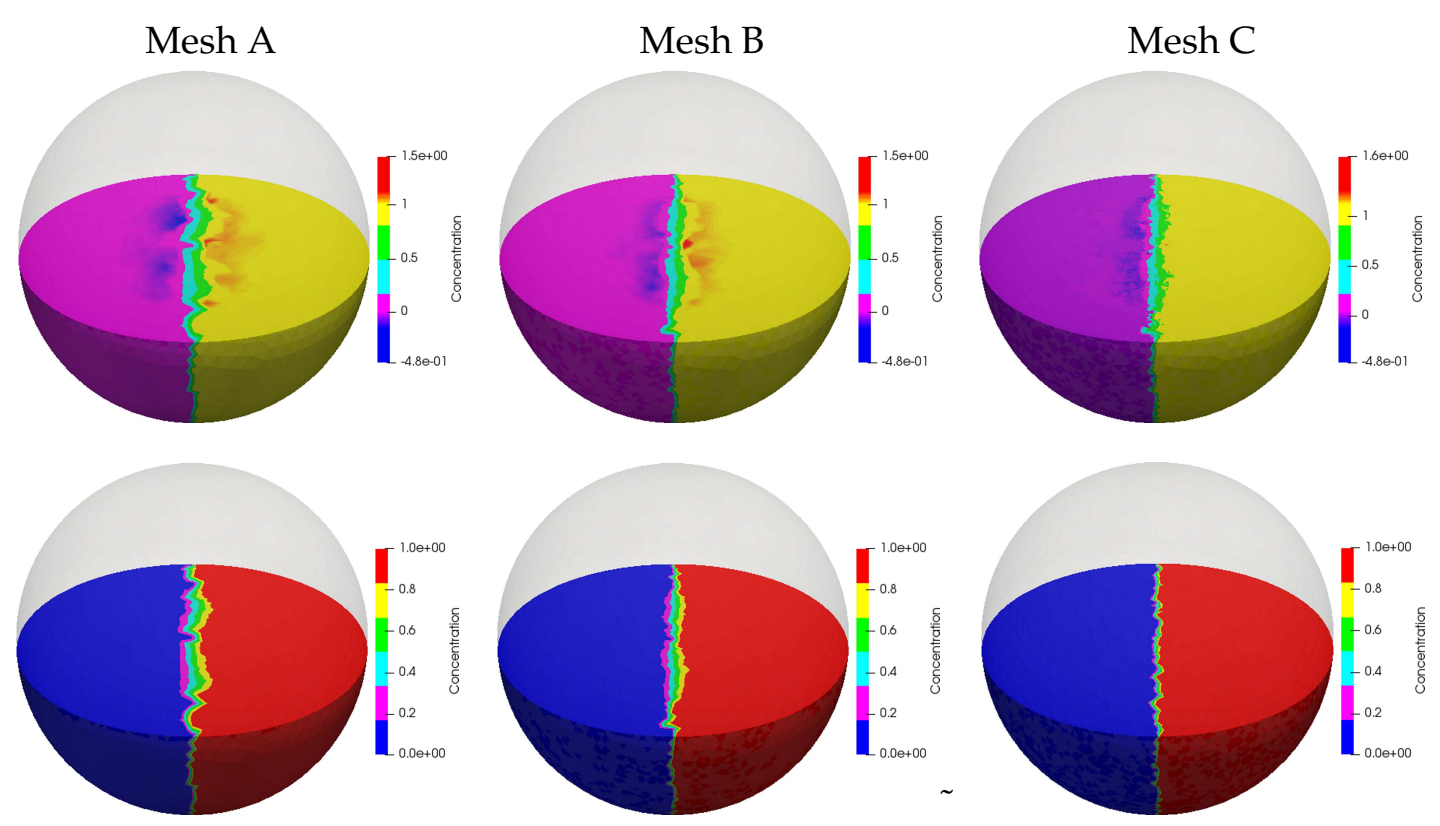

Figure 9: Same as Fig. 8 but at time $t=T$. 

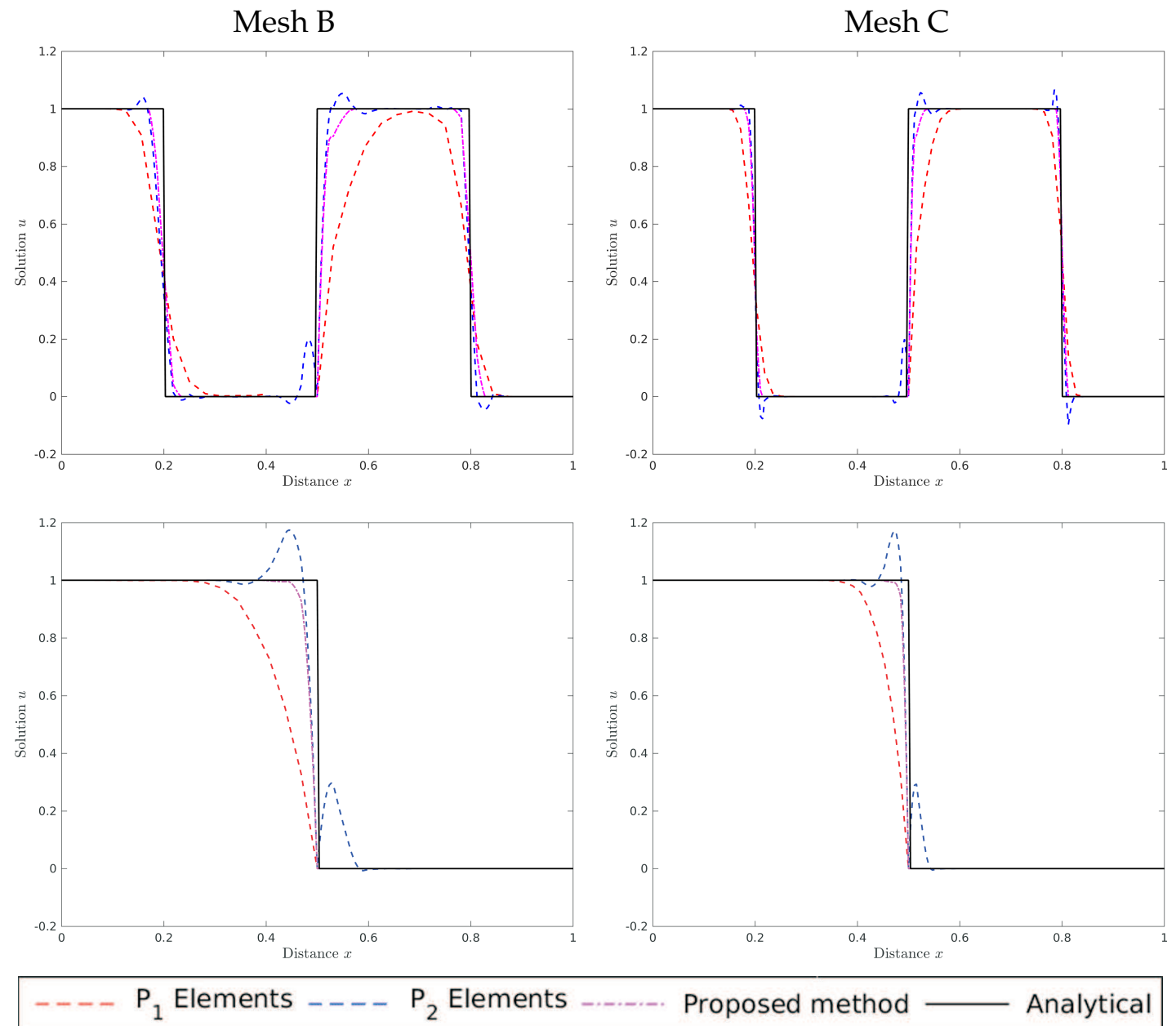

Figure 10: Cross-sections of the solutions at $y=0.25$ and $z=0.5$ for the deformational flow problem on Mesh $\mathrm{B}$ (first column) and Mesh C (second column) at time $t=\frac{T}{2}$ (first row) and $t=T$ (second row).

$y=0.25$ and $z=0.5$ of the computed results using Mesh B and Mesh C at times $t=\frac{T}{2}$ and $t=T$. In this figure wee also include results obtained using the linear $P_{1}$ elements. Obviously, the resolution and location of the interface are deteriorated with the large numerical diffusion generated by the linear $P_{1}$ elements. As expected, the method using the quadratic $P_{2}$ elements has managed to eliminate the numerical diffusion, but it yields non-physical oscillations near regions of large gradients in the computational domain. On the other hand, comparing the performance of proposed method in Fig. 10, nearly identical results to those obtained using the quadratic $P_{2}$ elements in regions with smooth gradients are achieved and a negligible numerical diffusion is introduced compared to the one generated by the linear $P_{1}$ elements. It is also clear that solutions obtained using the proposed method eliminate the non-physical oscillations near the interface where 
Table 3: Results for the deformational flow at $t=T$ and $t=\frac{T}{2}$. The exact maximum and minimum are respectively $\operatorname{Max}=1$ and $\operatorname{Min}=0$.

\begin{tabular}{|c|c|c|c|c|c|c|c|c|}
\hline \multirow[b]{3}{*}{ Mesh } & \multicolumn{7}{|c|}{$P_{1}$ Elements } & \\
\hline & \multicolumn{4}{|c|}{$t=\frac{T}{2}$} & \multicolumn{4}{|c|}{$t=T$} \\
\hline & Min & Max & Mass & CPU & Min & Max & Mass & CPU \\
\hline Mesh A & 0.000 & 1.000 & 0.973 & 0.063 & 0.000 & 1.000 & 0.949 & 0.117 \\
\hline Mesh B & 0.000 & 1.000 & 0.989 & 0.393 & 0.000 & 1.000 & 0.979 & 0.745 \\
\hline Mesh C & 0.000 & 1.000 & 0.996 & 2.794 & 0.000 & 1.000 & 0.992 & 5.246 \\
\hline \multicolumn{9}{|c|}{$P_{2}$ Elements } \\
\hline & \multicolumn{4}{|c|}{$t=\frac{T}{2}$} & \multicolumn{4}{|c|}{$t=T$} \\
\hline Mesh & Min & Max & Mass & $\mathrm{CPU}$ & Min & Max & Mass & CPU \\
\hline Mesh A & -0.153 & 1.663 & 0.965 & 0.399 & -0.112 & 1.423 & 0.942 & 0.730 \\
\hline Mesh B & -0.208 & 1.664 & 0.982 & 2.693 & -0.186 & 1.512 & 0.965 & 5.047 \\
\hline Mesh C & -0.184 & 1.664 & 0.998 & 20.812 & -0.178 & 1.499 & 0.994 & 38.813 \\
\hline \multicolumn{9}{|c|}{ Proposed method } \\
\hline & \multicolumn{4}{|c|}{$t=\frac{T}{2}$} & \multicolumn{4}{|c|}{$t=T$} \\
\hline Mesh & Min & Max & Mass & $\overline{\mathrm{CPU}}$ & Min & $\operatorname{Max}$ & Mass & $\overline{\mathrm{CPU}}$ \\
\hline Mesh A & 0.000 & 1.000 & 1.000 & 0.414 & 0.000 & 1.000 & 1.000 & 0.772 \\
\hline Mesh B & 0.000 & 1.000 & 1.000 & 2.865 & 0.000 & 1.000 & 1.000 & 5.374 \\
\hline Mesh C & 0.000 & 1.000 & 1.000 & 22.397 & 0.000 & 1.000 & 1.000 & 43.693 \\
\hline
\end{tabular}

discontinuities are steep. Thus, for the considered transport conditions, the deformational flow is accurately resolved without requiring fine meshes or small time steps in the simulations.

We now turn our attention to a quantitative comparison of the results using the linear $P_{1}$ elements, quadratic $P_{2}$ elements and proposed conservative method for the three considered meshes at times $t=\frac{T}{2}$ and $t=T$. In Table 3 we present the minimum (Min) and the maximum (Max) values of the computed solutions, the relative mass (Mass) and the CPU times for each simulation. From the values of Min and Max, we observe large and negative values for the quadratic $P_{2}$ elements which are completely avoided in our conservative and monotone method. Regarding the mass conservation, Table 3 reveals that on Mesh B, the quadratic $P_{2}$ elements lost more than $3 \%$ of the initial mass at time $t=T$, whereas the proposed characteristic finite element method is conservative at the machine precision. This clearly demonstrates that the proposed limiting procedure does not deteriorate the accuracy of the characteristic finite element method. Furthermore, a simple examination of the CPU time in Table 3 confirms that, on coarse meshes, there is no huge difference between the computational cost required for the quadratic $P_{2}$ elements and the proposed method. Again, in all results summarized in Table 3, the CPU time needed for the proposed conservative characteristic finite element method is about 
$17 \%$ more than the CPU time needed for the quadratic $P_{2}$ elements. It is worth remarking that the extra computational cost used in the proposed conservative and monotone procedures has been kept to the minimum that the characteristic finite element method is still effective for this class of convection-dominated flow problems. Taking all these factors into account, we conclude that, for the considered examples, the proposed characteristic finite element method exhibits higher monotone and non-oscillatory properties than the standard linear $P_{1}$ and quadratic $P_{2}$ elements. More importantly, a balance between efficiency and accuracy in these methods benefits the conservative and monotone method, since the additional cost required for the limiting procedure in characteristic finite element method is minimal while the results obtained by the method are fully conservative and more accurate than those obtained by the quadratic $P_{2}$ elements. Therefore, hereafter we shall focus our attention on numerical simulations carried out using only the proposed conservative and monotone method.

\subsection{Flow past a circular cylinder}

To assess the numerical performance of the proposed characteristic finite element method for solving three-dimensional incompressible Navier-Stokes equations we consider the benchmark problem of the flow past a circular cylinder. This flow problem has been widely used in the literature to examine the accuracy of numerical methods for incompressible Navier-Stokes equations, see for example [1,14,31,37]. In our simulations presented in this section, we use the same flow configuration and the same boundary conditions as those reported in these references. Here, circular cylinder with diameter $D=0.1$ is immersed in a channel with height $H=0.41$ subject to a viscous incompressible flow entering the channel with a parabolic velocity defined as

$$
u(t, y, z)=u_{\infty} y(H-y) z(H-z) H^{4} \sin \left(\frac{\pi t}{8}\right)
$$

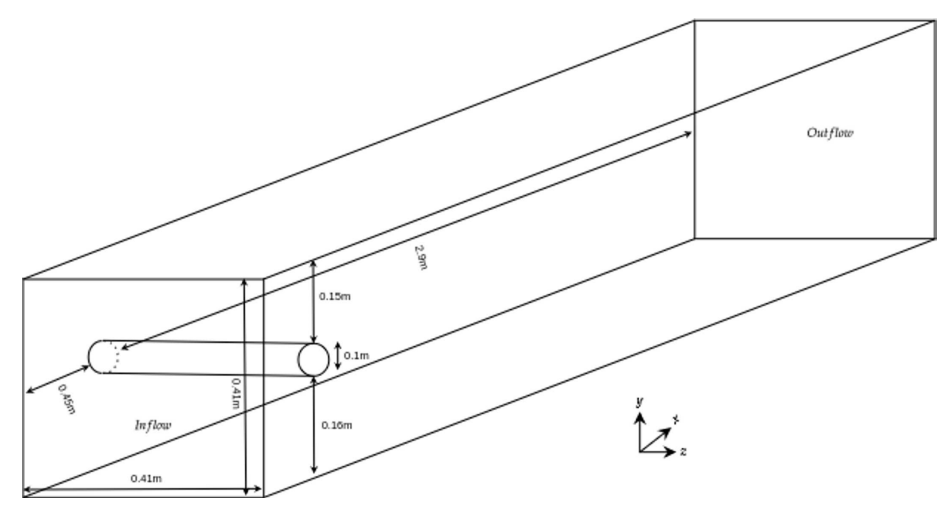

Figure 11: Configuration of the computational domain used for the flow past a circular cylinder. 

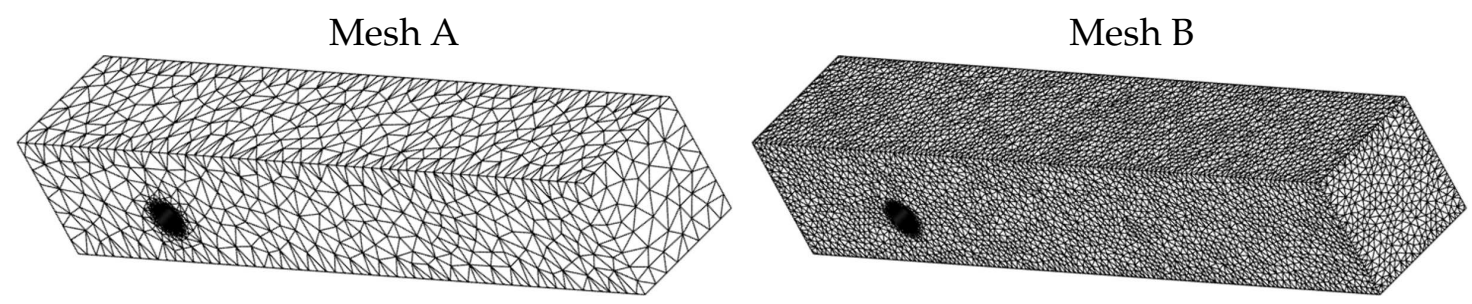

Figure 12: Computational meshes used in simulations for the flow past a circular cylinder.

where $u_{\infty}=7.2$. The Reynolds number for this flow problem is defined as $R e=D u_{\infty} / v$, with $v$ is the kinematic viscosity. At the downstream boundary we impose the pseudostress condition

$$
-p n+v \frac{\partial u}{\partial n}=0,
$$

where $\boldsymbol{n}=\left(n_{x}, n_{y}, n_{z}\right)^{\top}$ is the outward unit normal on the exit boundary. On the remaining boundaries of the computational domain we set no-slip conditions $\boldsymbol{u}=\mathbf{0}$. All the computations for this flow problem are carried out using the mixed tetrahedral finite elements $P_{1}-P_{2}$ using two unstructured meshes depicted in Fig. 12 and numerical results are presented at time $t=8$ for two different Reynolds numbers $R e=20$ and $R e=100$ using a fixed time step $\Delta t=0.1$. At these two values of Reynolds number, the flow is expected to illustrate different flow features and the problem becomes challenging for high values of $R e$. Note that most of results reported in the literature considered only the case with $R e=20$.

First a mesh convergence study for the proposed characteristic finite element method is performed for this example. To this end, we consider four unstructured meshes Mesh A, Mesh B, Mesh C and Mesh D with different node and element densities as summarized in Table 4. Note that Mesh C and Mesh D are not included in Fig. 12 because of their densities which result in heavily black plots. In Table 4 we also summarize the maximum and minimum values of the velocity $u$ along with the CPU times obtained using both the conventional and the proposed methods on the considered meshes for $R e=20$ and $R e=100$. As can be seen for the last two mesh levels Mesh C and Mesh D, the differences in the values obtained for $\max u$ and $\min u$ are very small. It has also been observed that the additional computational effort used by the proposed limiting procedure has been kept to the minimum that our characteristic finite element method is still competitive for three-dimensional incompressible Navier-Stokes equations. It should be stressed that the vast amount of the computational work is mainly used for solving the linear systems in the Stokes stage of the conjugate gradient algorithm. Hence, a substantial reduction in the CPU time can be achieved in the proposed method by developing more efficient preconditioned iterative solvers for these linear systems. For instance, multigrid techniques are well known to be the most efficient methods for solving linear systems and can therefore be the suitable tools to increase the efficiency of the proposed characteristic finite element method. To further qualify the computational results for these meshes, Fig. 13 
Table 4: Mesh statistics and results for maximum and minimum of the velocity $u$ and CPU times (in minutes) for the flow past a circular cylinder at $R e=20$ and $R e=100$.

\begin{tabular}{|c|c|c|c|c|c|c|c|}
\hline \multirow[b]{3}{*}{ Mesh } & \multirow[b]{3}{*}{ \# elements } & \multicolumn{6}{|c|}{$R e=20$} \\
\hline & & \multicolumn{3}{|c|}{ Conventional method } & \multicolumn{3}{|c|}{ Proposed method } \\
\hline & & $\max u$ & $\min u$ & $\mathrm{CPU}$ & $\max u$ & $\min u$ & $\mathrm{CPU}$ \\
\hline Mesh A & 114446 & 0.21420 & $-3.6639 \mathrm{E}-02$ & 38.9 & 0.20456 & $-3.3191 E-04$ & 39.4 \\
\hline Mesh B & 195930 & 0.20840 & $-3.5239 \mathrm{E}-03$ & 118.4 & 0.20466 & $-3.3163 \mathrm{E}-04$ & 118.9 \\
\hline Mesh C & 301436 & 0.20701 & $-1.9251 \mathrm{E}-03$ & 158.8 & 0.20468 & $-3.2151 \mathrm{E}-04$ & 162.7 \\
\hline \multirow[t]{3}{*}{ Mesh D } & 436327 & 0.20531 & -8.9154E-04 & 243.3 & 0.20468 & $-3.2150 \mathrm{E}-04$ & 243.5 \\
\hline & & \multicolumn{6}{|c|}{$R e=100$} \\
\hline & & \multicolumn{3}{|c|}{ Conventional method } & \multicolumn{3}{|c|}{ Proposed method } \\
\hline Mesh & \# elements & $\max u$ & $\min u$ & $\mathrm{CPU}$ & $\max u$ & $\min u$ & $\mathrm{CPU}$ \\
\hline Mesh A & 114446 & 0.22327 & $-4.4228 \mathrm{E}-02$ & 31.8 & 0.20523 & $-2.5217 \mathrm{E}-03$ & 32.9 \\
\hline Mesh B & 195930 & 0.21299 & $-1.8903 \mathrm{E}-03$ & 91.3 & 0.20625 & $-1.6123 \mathrm{E}-04$ & 91.6 \\
\hline Mesh C & 301436 & 0.21012 & $-5.1163 \mathrm{E}-04$ & 135.0 & 0.20635 & $-1.6113 \mathrm{E}-04$ & 135.7 \\
\hline Mesh D & 436327 & 0.20497 & $-2.8145 \mathrm{E}-04$ & 241.9 & 0.20635 & $-1.6113 \mathrm{E}-04$ & 243.1 \\
\hline
\end{tabular}
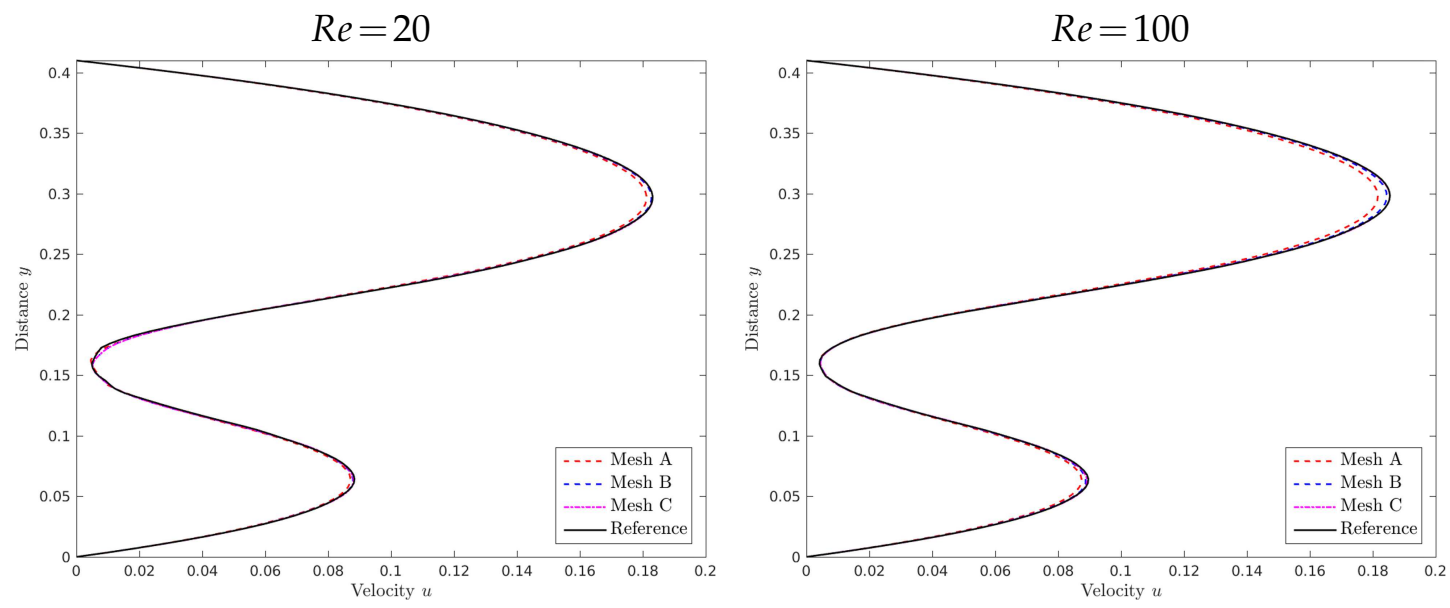

Figure 13: Cross-sections of the velocity $u$ at $(x=0.56, z=0.25)$ obtained on different meshes for the flow past a circular cylinder at $R e=20$ (left) and $R e=100$ (right).

illustrates the cross-sections of the velocity $u$ at the line $(x=0.56, z=0.25)$ obtained using the considered meshes. It is clear that increasing the density of meshes, the results for the Mesh C and Mesh D are relatively similar. Results obtained for the velocities $v$ and $w$, not reported here for brevity, exhibit the same convergence features. Therefore, Mesh A and Mesh B are used in all our next computations for this flow problem. Note that the reasons for choosing these mesh structures lie essentially on the computational cost required for each mesh configuration and also on the numerical resolution achieved. 


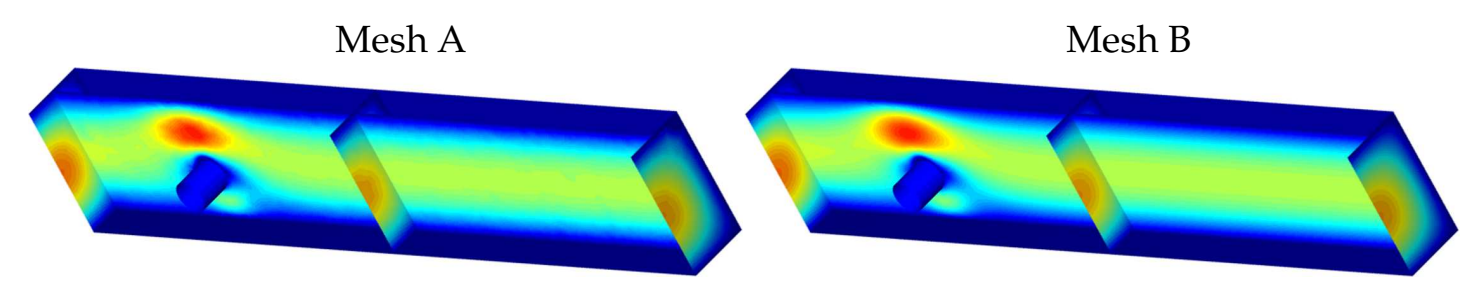

Figure 14: Snapshots of the velocity magnitude for the flow past a circular cylinder at $R e=100$ obtained on Mesh A (left) and Mesh B (right).

In Fig. 14 we display the obtained results for the magnitude of the velocity field using $R e=100$ on Mesh A and Mesh B. For a better insight, only a part of the computational domain is shown in this figure. These plots give a clear view of the overall flow patterns and the effect of the mesh on the velocity field. The recirculating regions behind the cylinder are generated and well captured using the proposed method. It is also clear that the performance of the proposed characteristic finite element method is very attractive since the obtained numerical solutions are stable and monotone even when coarse meshes are used with no need to nonlinear solvers or complicated techniques to stabilize the finite element discretization as those used in the projection methods or pressure correction procedures. Comparisons between results obtained using the proposed method and the conventional characteristic finite element method have also been carried out in this problem. Fig. 15 depicts cross-sections of the velocity $u$ at the line $(x=0.56, z=0.25)$ obtained using the proposed and conventional methods on three different meshes at $R e=20$ and $R e=100$. It is clear that, on Mesh A and Mesh B, the conventional method exhibits substantially greater oscillations, specially for $y \in[0.25,0.35]$ in the spatial domain where the velocity $u$ is large. From the same figure we observe a complete absence of this oscillatory behavior in the results obtained using the proposed method. Note that the relevance of the monotonicity and non-oscillatory properties for this problem is crucial since these oscillations are physically unacceptable and having non-monotone solutions may cause a breakdown in the iterations of the conjugate gradient solver used to solve the linear systems for the Stokes stage in the conventional algorithm. On the fine mesh Mesh C, results obtained using both method coincide for $R e=20$ but for $R e=100$ the numerical diffusion in the results obtained the conventional method is clearly visible. On the contrary, computational results obtained using the proposed method are free from any non-physical oscillations, and the solutions preserve the monotonicity during the time integration process.

To quantify our results for this flow problem, we compute the well-established lift and drag coefficients at each time step as [1]

$$
\begin{aligned}
& C_{L}=-\frac{2}{u_{\infty}^{2} D H} \oint_{S}\left(-p n_{x}+v \frac{\partial(\boldsymbol{u} \cdot \mathbf{t})}{\partial \mathbf{n}} n_{y}\right) d x, \\
& C_{D}=\frac{2}{u_{\infty}^{2} D H} \oint_{S}\left(-p n_{y}+v \frac{\partial(\boldsymbol{u} \cdot \mathbf{t})}{\partial \mathbf{n}} n_{x}\right) d x,
\end{aligned}
$$

where $S$ is the surface of the cylinder, $\mathbf{n}=\left(n_{x}, n_{y}, 0\right)^{\top}$ and $\mathbf{t}=\left(n_{y},-n_{x}, 0\right)^{\top}$ are respectively 

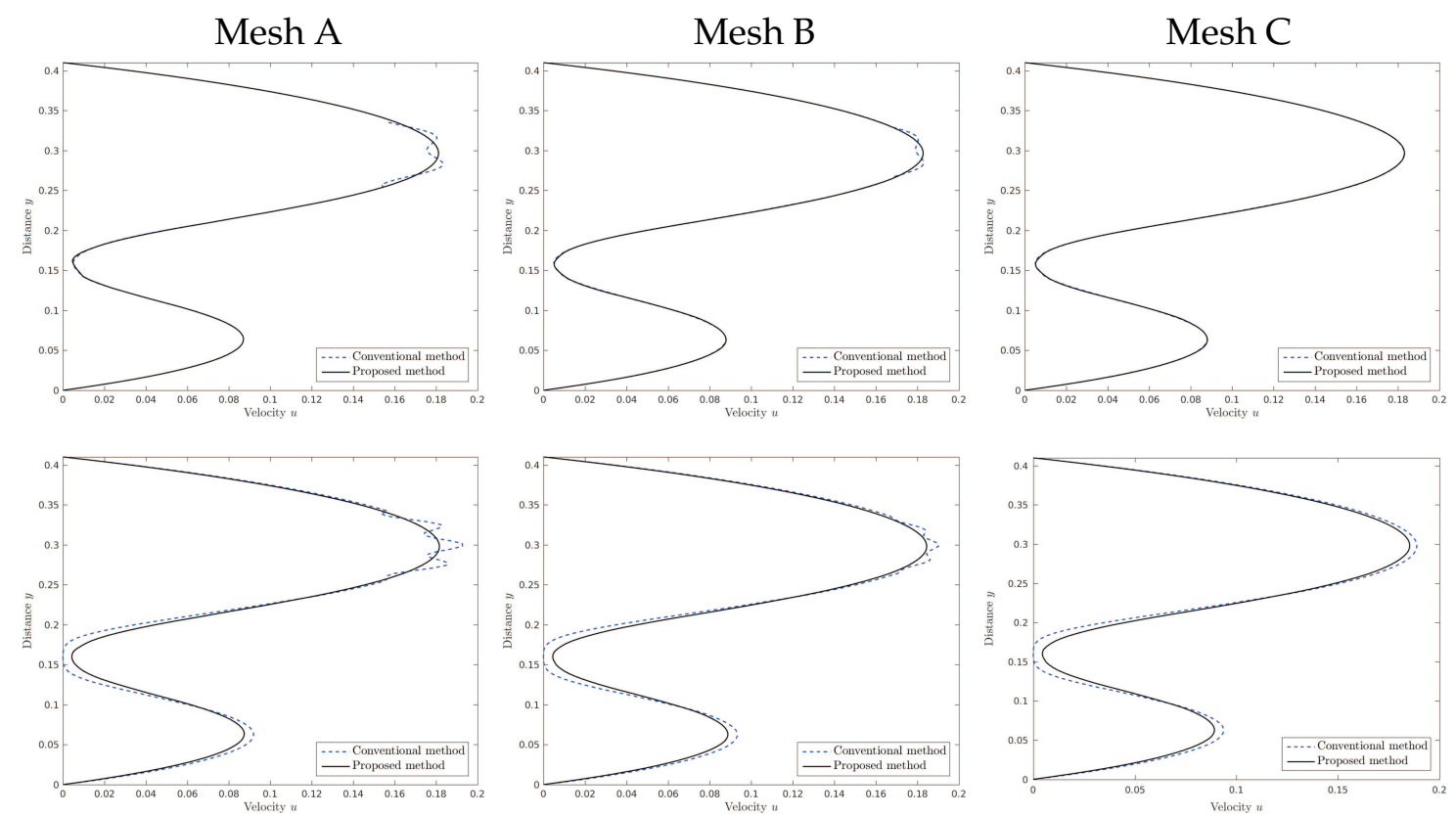

Figure 15: Cross-sections of the velocity $u$ at $(x=0.56, z=0.25)$ obtained on three different meshes for the flow past a circular cylinder at $R e=20$ (first row) and $R e=100$ (second row).

Table 5: Comparison results for mesh statistics, lift and drag coefficients, average iterations of the conjugate gradient algorithm and CPU times (in minutes) for the flow past a circular cylinder at $R e=20$.

\begin{tabular}{||lcccccc||}
\hline Mesh & \# elements & $\max _{D}$ & $\max C_{L}$ & $\min C_{L}$ & AvCG & CPU \\
\hline Mesh A & 114446 & 3.235 & 0.0271 & $-1.0131 \mathrm{E}-02$ & 3.45 & 39.4 \\
Mesh B & 195930 & 3.301 & 0.0274 & $-1.0981 \mathrm{E}-02$ & 3.21 & 118.9 \\
Mesh C & 301436 & 3.301 & 0.0279 & $-1.0991 \mathrm{E}-02$ & 3.14 & 162.7 \\
Mesh D & 436327 & 3.301 & 0.0280 & $-1.0992 \mathrm{E}-02$ & 3.11 & 243.5 \\
Results from [1] & 393216 & 3.296 & 0.0280 & $-1.0992 \mathrm{E}-02$ & - & - \\
\hline
\end{tabular}

the normal and tangent unit vectors with respect to the surface of the cylinder. Results obtained for $\max C_{D}, \max C_{L}, \min C_{L}$, the number of averaged iterations in the conjugate gradient algorithm (AvCG) and the computational cost (CPU) are summarized in Table 5. We present numerical results obtained for $R e=20$ using the four considered meshes and for comparison purpose, we also include those numerical results reported in [1]. The numerical results obtained using the proposed method mostly agree with all the model results reported in [1]. As it is obvious from Table 5, the minor differences to other numerical results can be attributed to the mesh size used in the present study. The efficiency of the proposed characteristic finite element method is also examined for this problem. Note that the computational time listed in Table 5 includes all aspects of computational work including, mesh generation, calculation of characteristics, search-locate of depar- 
Table 6: Percentage of the computational cost used by each stage of the conventional and the proposed characteristic finite element solvers for the flow past a circular cylinder at $R e=20$ and $R e=100$ using Mesh $A$. The CPU times are given in minutes.

\begin{tabular}{|c|c|c|c|c|c|}
\hline \multirow[b]{3}{*}{ Problem } & \multirow[b]{3}{*}{ Algorithm } & \multicolumn{4}{|c|}{$R e=20$} \\
\hline & & \multicolumn{2}{|c|}{ Conventional method } & \multicolumn{2}{|c|}{ Proposed method } \\
\hline & & $\mathrm{CPU}$ & Percentage & CPU & Percentage \\
\hline \multirow{4}{*}{ Convection part } & Departure points & 2.83 & $7.22 \%$ & 2.86 & $7.26 \%$ \\
\hline & Search-locate & 2.70 & $6.89 \%$ & 2.98 & $7.57 \%$ \\
\hline & Interpolation & 2.12 & $5.41 \%$ & 3.31 & $8.41 \%$ \\
\hline & Total & 7.65 & $19.52 \%$ & 9.15 & $23.24 \%$ \\
\hline Stokes part & CG solver & 31.55 & $80.48 \%$ & 30.23 & $76.76 \%$ \\
\hline \multicolumn{2}{|l|}{ Total } & 38.80 & $100 \%$ & 39.38 & $100 \%$ \\
\hline \multirow[b]{3}{*}{ Problem } & \multirow[b]{3}{*}{ Algorithm } & \multicolumn{4}{|c|}{$R e=100$} \\
\hline & & \multicolumn{2}{|c|}{ Conventional method } & \multicolumn{2}{|c|}{ Proposed method } \\
\hline & & CPU & Percentage & CPU & Percentage \\
\hline \multirow{4}{*}{ Convection part } & Departure points & 2.17 & $6.82 \%$ & 2.02 & $6.14 \%$ \\
\hline & Search-locate & 2.31 & $7.27 \%$ & 2.61 & $7.95 \%$ \\
\hline & Interpolation & 2.27 & $7.14 \%$ & 3.56 & $10.83 \%$ \\
\hline & Total & 6.75 & $21.23 \%$ & 8.19 & $24.92 \%$ \\
\hline Stokes part & CG solver & 25.05 & $78.77 \%$ & 24.70 & $75.08 \%$ \\
\hline Total & & 31.80 & $100 \%$ & 32.89 & $100 \%$ \\
\hline
\end{tabular}

ture points, limiting process, assembling of finite element matrices, and solution of linear systems. In our implementation, most of the computational effort was contributed to the conjugate gradient algorithm used for solving the associated linear systems. For this flow problem, we have observed that the mean number of iterations in the conjugate gradient algorithm to converge to a tolerance of $10^{-7}$ increases with the values of the Reynolds number Re. Nevertheless, it should be noted that the proposed characteristic finite element method can use time steps up to hundred times larger than those required for the explicit time integration schemes. This would allow for fewer time steps to be taken for the same length of simulation, thereby reducing the overall computational cost excessively.

Analysis of computational cost has been carried out in this example. Table 6 summarizes the computational cost of the conventional and the proposed characteristic finite element methods at $R e=20$ and $R e=100$ using Mesh A. The computational cost is distributed in four stages constituting the proposed method: Departure points represents the percent of CPU time involved in the approximation of departure points. Search-locate denotes the percent of CPU allocated for the search-locate procedure. Interpolation refers the percent of CPU time required for the interpolation procedure used in the method. CG 
refers to the percent of CPU time employed in the conjugate gradient algorithm for solving the Stokes problem. The main features reported in this table are on one hand, the departure points approximations, interpolation stage, and the search-locate procedure require very little computational cost compared to the CPU time needed for the Stokes part where the linear solver is repeatedly required. On the other hand, the difference between the CPU times spent in the convection part for the conventional and the proposed monotone and conservative methods is minimal and does not exceed $18 \%$ in all considered cases. As can be seen from Table 6, most of the computational effort goes into the CG algorithm solving the associated Stokes problems. Therefore, reducing the CPU time in the proposed characteristic finite element method can be achieved by constructing more efficient preconditioned iterative solvers for the linear systems. Multigrid techniques are known to be the most efficient methods for solving linear systems and can therefore be the suitable tools to increase the efficiency of the proposed characteristic finite element method. Needless to say that the CPU time in Table 6 can drastically be reduced if parallel computers are used. A parallel characteristic finite element method can be implemented using the message passing interface (MPI). The parallel efficiency using the MPI becomes very high when the departure points lie within the local memory of the same processor and the operation per processor is kept constant.

\section{Concluding remarks}

A conservative and monotone characteristic finite element method has been developed for solving the three-dimensional transport and incompressible Navier-Stokes equations on unstructured tetrahedral meshes. The proposed method integrates transport part of the equations using the modified method of characteristics in the framework of a finite element discretization. To preserve the conservation and monotonicity in the proposed method a local limiting procedure is implemented using the linear and quadratic finite elements. The additional computational cost in the proposed characteristic finite element method is kept minimal compared to its conventional counterparts. Using a mixed finite element formulation the method is applied for the three-dimensional incompressible Navier-Stokes equations in primitive variables. We also implemented a direct conjugategradient algorithm for the solution of the three-dimensional Stokes problem. This algorithm avoids projection techniques and special corrections for the pressure widely used in the literature for Eulerian-based finite element methods. Numerical results were presented for the problem of rotating a slotted sphere in circular flow fields and the example of a deformational flow problem. The proposed method has also been verified for the numerical simulation of the benchmark problem of flow past a circular cylinder. For all of these examples, the obtained results have demonstrated the ability of the proposed characteristic finite element method to perform very well in the presence of strong gradients and discontinuities without non-physical oscillations and numerical dissipation even when coarse meshes and large time steps are used in the simulations. Results ob- 
tained for the problem of flow past a circular cylinder have also shown the capabilities of the proposed method in simulations of complex incompressible flow problems in three space dimensions using unstructured tetrahedral meshes. Future work will concentrate on the extension of the conservative and monotone characteristic finite element method to coupled problems of turbulent flow and heat transfer in three space dimensions.

\section{Acknowledgments}

Financial support provided by the project CSIC under the contract MTM2017-89423-P is gratefully acknowledged.

\section{References}

[1] E. Bayraktar, O. Mierka, and S. Turek. Benchmark computations of 3D laminar flow around a cylinder with CFX, OpenFOAM and FeatFlow. International Journal of Computational Science and Engineering, 7:253-266, 2012.

[2] R. Bermejo. Analysis of a class of quasi-monotone and conservative semi-Lagrangian advection schemes. Numerische Mathematik, 87:597-623, 2001.

[3] F. Bertrand, L. Demkowicz, J. Gopalakrishnan, and N. Heuer. Recent advances in leastsquares and discontinuous Petrov-Galerkin finite element methods. Computational Methods in Applied Mathematics, 19:395-397, 2019.

[4] Sh. Boroun and F. Larachi. Anomalous anisotropic transport of scalars in dilute ferrofluids under uniform rotating magnetic fields-mixing time measurements and ferrohydrodynamic simulations. Chemical Engineering Journal, 380:122504, 2020.

[5] A.N. Brooks and T.JR. Hughes. Streamline upwind/Petrov-Galerkin formulations for convection dominated flows with particular emphasis on the incompressible Navier-Stokes equations. Computer Methods in Applied Mechanics and Engineering, 32:199-259, 1982.

[6] X. Cai, J. Qiu, and J.-M. Qiu. A conservative semi-Lagrangian HWENO method for the Vlasov equation. J. Computational Physics, 323:95-114, 2016.

[7] A. Cameron. A systematic method to enforce conservativity on semi-Lagrangian schemes. Int. J. Numer. Methods. Fluids., 88:463-478, 2018.

[8] E.O.A. Carew, P. Townsend, and M.F. Webster. A Taylor-Petrov-Galerkin algorithm for viscoelastic flow. Journal of Non-Newtonian Fluid Mechanics, 50:253-287, 1993.

[9] Y. Chai, Z. Gong, W. Li, T. Li, Q. Zhang, Z. Zou, and Y. Sun. Application of smoothed finite element method to two-dimensional exterior problems of acoustic radiation. International Journal of Computational Methods, 15:1850029, 2018.

[10] H. Chen, K. Li, and S. Wang. A dimension split method for the incompressible Navier-Stokes equations in three dimensions. Int. J. Numer. Methods. Fluids., 73(5):409-35, 2013.

[11] M.J. Crochet and V. Legat. The consistent streamline-upwind/Petrov-Galerkin method for viscoelastic flow revisited. Journal of Non-NEwtonian Fluid Mechanics, 42:283-299, 1992.

[12] X.Y. Cui, S. Chang, and G.Y. Li. A two-step Taylor-Galerkin smoothed finite element method for Lagrangian dynamic problem. International Journal of Computational Methods, 12:1540004, 2015.

[13] M. Dehghan. Numerical solution of the three-dimensional advection-diffusion equation. Applied Mathematics and Computation, 150:5-19, 2004. 
[14] S. Deparis, M.O. Deville, F. Menghini, L. Pegolotti, and A. Quarteroni. Application of the Rosenbrock methods to the solution of unsteady 3D incompressible Navier-Stokes equations. Computers \& Fluids, 179:112-122, 2019.

[15] S. Deparis and A.E. Løvgren. Stabilized reduced basis approximation of incompressible three-dimensional Navier-Stokes equations in parametrized deformed domains. Journal of Scientific Computing, 50:198-212, 2012.

[16] J. Donea. A Taylor-Galerkin method for convective transport problems. Int. J. Numer. Meth. Engng., 20:101-119, 1984.

[17] J. Douglas and T.F. Russell. Numerical methods for convection dominated diffusion problems based on combining the method of characteristics with finite elements or finite differences. SIAM Journal on Numerical Analysis, 19:871-885, 1982.

[18] M. El-Amrani and M. Seaid. Convergence and stability of finite element modified method of characteristics for the incompressible Navier-Stokes equations. Journal of Numerical Mathematics, 15:101-135, 2007.

[19] M. El-Amrani and M. Seaid. Numerical simulation of natural and mixed convection flows by Galerkin-characteristic method. Int. J. Numer. Meth. Fluids., 53:1819-1845, 2007.

[20] M. El-Amrani and M. Seaid. A finite element modified method of characteristics for convective heat transport. Numerical Methods for Partial Differential Equations, 24:776-798, 2008.

[21] M. El-Amrani and M. Seaid. An essentially non-oscillatory semi-Lagrangian method for tidal flow simulations. Int. J. Numer. Meth. Engng., 81:805-834, 2010.

[22] M. El-Amrani and M. Seaid. An L ${ }^{2}$-projection for the Galerkin-characteristic solution of incompressible flows. SIAM Journal on Scientific Computing, 33:3110-3131, 2011.

[23] M. El-Amrani and M. Seaid. A conjugate gradient algorithm for solving the Galerkincharacteristic approximation of interfacial flows. Applied Numerical Mathematics, 62:1197$1214,2012$.

[24] M. El-Amrani and M. Seaid. A finite element semi-Lagrangian method with $\mathrm{L}^{2}$ interpolation. Int. J. Numer. Meth. Engng., 90:1485-1507, 2012.

[25] M. Hedayati-Dezfooli and W.H. Leong. An experimental study of coupled heat and moisture transfer in soils at high temperature conditions for a medium coarse soil. International Journal of Heat and Mass Transfer, 137:372-389, 2019.

[26] T. JR. Hughes, L. P. Franca, and G. M. Hulbert. A new finite element formulation for computational fluid dynamics: VIII. The Galerkin/least-squares method for advective-diffusive equations. Computer Methods in Applied Mechanics and Engineering, 73:173-189, 1989.

[27] L.A. Khan and P.L.F. Liu. An operator splitting algorithm for the three-dimensional advection-diffusion equation. Int. J. Numer. Methods. Fluids., 28:461-476, 1998.

[28] B. Khouya, M. El-Amrani, and M. Seaid. A Galerkin-characteristic finite element method for three-dimensional convection-dominated problems. Adv. Appl. Math. Mech., 13:503-526, 2021.

[29] B. Khouya, M. El-Amrani, and M. Seaid. A three-dimensional monotonicity-preserving modified method of characteristics on unstructured tetrahedral meshes. International Journal of Computational Methods, page 2050027, 2020.

[30] R.J. LeVeque. High-resolution conservative algorithms for advection in incompressible flow. SIAM Journal on Numerical Analysis, 33:627-665, 1996.

[31] Z. Li, M.A. Prsic, M. C. Ong, and B. C. Khoo. Large Eddy simulations of flow around two circular cylinders in tandem in the vicinity of a plane wall at small gap ratios. Journal of Fluids and Structures, 76:251-271, 2018.

[32] C.H. Liu. Numerical solution of three-dimensional Navier-Stokes equations by a velocity- 
vorticity method. Int. J. Numer. Methods. Fluids., 35:533-557, 2001.

[33] D.C. Lo, K. Murugesan, and D.L. Young. Numerical solution of three-dimensional velocityvorticity Navier-Stokes equations by finite difference method. Int. J. Numer. Methods. Fluids., 47:1469-1487, 2005.

[34] Z. Ma, Y. Zhang, and Z. Zhou. An improved semi-Lagrangian time splitting spectral method for the semi-classical Schrödinger equation with vector potentials using NUFFT. Applied Numerical Mathematics, 111:144-159, 2017.

[35] N.S. Park and J. A. Liggett. Application of Taylor-least squares finite element to threedimensional advection-diffusion equation. Int. J. Numer. Methods. Fluids., 13:759-773, 1991.

[36] Z.S. Qian and J.B. Zhang. Implicit preconditioned high-order compact scheme for the simulation of the three-dimensional incompressible Navier-Stokes equations with pseudocompressibility method. Int. J. Numer. Methods. Fluids., 69:1165-1185, 2012.

[37] M. Schäfer, S. Turek, F. Durst, E. Krause, and R. Rannacher. Benchmark computations of laminar flow around a cylinder. In Flow Simulation with High-Performance Computers II, pages 547-566. 1996.

[38] M. Seaid. On the quasi-monotone modified method of characteristics for transport-diffusion problems with reactive sources. Comput. Methods Appl. Math., 2:186-210, 2001.

[39] M. Seaid. Semi-Lagrangian integration schemes for viscous incompressible flows. Comput. Methods Appl. Math., 2:392-409, 2002.

[40] M. Seaid. Multidimensional relaxation approximations for hyperbolic systems of conservation laws. Journal of Computational Mathematics, pages 440-457, 2007.

[41] C.J. Subich, K.G. Lamb, and M. Stastna. Simulation of the Navier-Stokes equations in three dimensions with a spectral collocation method. Int. J. Numer. Methods. Fluids., 73:103-129, 2013.

[42] L. L. Takacs. A two-step scheme for the advection equation with minimized dissipation and dispersion errors. Monthly Weather Review, 113:1050-1065, 1985.

[43] M.T. Wang and T.H. Sheu. On a compact mixed-order finite element for solving the threedimensional incompressible Navier-Stokes equations. Int. J. Numer. Methods. Fluids., 25:513$522,1997$.

[44] S.Ø. Wille. The three-dimensional prolonged adaptive unstructured finite element multigrid method for the Navier-Stokes equations. Int. J. Numer. Methods. Fluids., 25:371-392, 1997.

[45] Y. Xu. Similarity solution and heat transfer characteristics for a class of nonlinear convectiondiffusion equation with initial value conditions. Mathematical Problems in Engineering, 19, 2019.

[46] T. Yabe, R. Tanaka, T. Nakamura, and F. Xiao. An exactly conservative semi-Lagrangian scheme (CIP-CSL) in one dimension. Monthly Weather Review, 129:332-344, 2001.

[47] S.T. Zalesak. Fully multidimensional flux-corrected transport algorithms for fluids. Journal of Computational Physics, 31:335-362, 1979.

[48] Y. Zhai, X. Feng, and D. Lu. A novel method to deduce high-order compact difference scheme for the three dimensional semilinear convection-diffusion equation with variable coefficients. Numerical Heat Transfer, B 63 (5):425-455, 2013.

[49] Z. Zhang. Conservative finite-difference methods on general grids. SIAM Review, 39:367$369,1997$. 